University of Nebraska - Lincoln

DigitalCommons@University of Nebraska - Lincoln

2010

\title{
Validation of a model for static and dynamic recrystallization in metals
}

Arthur Brown

Sandia National Laboratories, Livermore, CA

Douglas Bammann

Mississippi State University, Mississippi State, MS

Follow this and additional works at: https://digitalcommons.unl.edu/usdoepub

Part of the Bioresource and Agricultural Engineering Commons

Brown, Arthur and Bammann, Douglas, "Validation of a model for static and dynamic recrystallization in metals" (2010). US Department of Energy Publications. 106.

https://digitalcommons.unl.edu/usdoepub/106

This Article is brought to you for free and open access by the U.S. Department of Energy at DigitalCommons@University of Nebraska - Lincoln. It has been accepted for inclusion in US Department of Energy Publications by an authorized administrator of DigitalCommons@University of Nebraska - Lincoln. 


\title{
Validation of a model for static and dynamic recrystallization in metals ${ }^{\text {is }}$
}

\author{
Arthur A. Brown ${ }^{\mathrm{a}, *}$, Douglas J. Bammann ${ }^{\mathrm{b}}$ \\ ${ }^{a}$ Multi-Physics Modeling and Simulation Department, Sandia National Laboratories, Livermore, CA 94551, United States \\ ${ }^{\mathrm{b}}$ Mechanical Engineering Department, Mississippi State University, Mississippi State, MS 39762, United States
}

\section{A R T I C L E I N F O}

\section{Article history:}

Received 14 December 2010

Received in final revised form 22 December

2011

Available online 3 January 2012

\section{Keywords:}

A. Microstructures

A. Dislocations

B. Elastic-viscoplastic material

Misorientation - nominated

B. Constitutive behavior

\begin{abstract}
A B S T R A C T
In this paper, modifications are proposed to a phenomenological plasticity model to account for the evolution of recrystallization and the resultant softening behavior. The novel model includes internal state variables representing dislocation density and the spacing between geometrically necessary subgrain boundaries. In order to capture both single and multiple peak recrystallization, the model tracks the evolution of recrystallized volume fractions for multiple cycles of recrystallization, and has a set of state variables for each volume fraction. A rule of mixtures is used to determine the average stress. The model is capable of capturing static recrystallization as well as both single and multiple peak dynamic recrystallization.

Material parameters are fit to data from monotonic compression tests on copper for a wide range of temperatures and strain rates. The model is then validated by using the same parameter set to predict multiple-stage response in which samples are compressed, held at temperature for various lengths of time, and then compressed further. The model predicts both the static recrystallization that occurs between loading stages as well as the dynamic recrystallization occurring during the second loading stage.
\end{abstract}

(c) 2012 Elsevier Ltd. All rights reserved.

\section{Introduction}

During high temperature manufacturing processes, metals undergo microstructural changes that can greatly affect material properties and residual stresses. Some of the physical mechanisms that influence the strength of a material are strain hardening, recovery, recrystallization, and grain growth (Kocks et al., 1975; Doherty et al., 1997). If the deformation conditions such as temperature and strain rate are not controlled properly during forging, welding, rolling, or other processes, the final part may have inadequate strength or residual stresses that could be detrimental to the life of the part (Chiesa et al., 2004). In order to be able to optimize manufacturing processes using computational capabilities, it is necessary to have a physically-based constitutive model that captures the dominant strengthening and softening mechanisms. Such a model with predictive capabilities can be used in an optimization scheme to reduce the number of design iterations required to produce a part that meets all strength and microstructural requirements.

Recrystallization is a complex, inhomogeneous process in which nucleation and growth of new strain-free grains replace the worked microstructure of a strained material (Haessner, 1978; Humphreys and Hatherly, 1995). Recrystallization is due to the motion of grain and subgrain boundaries. As the boundaries move, they sweep away the dislocation structure, leaving a strain-free material with a very low dislocation density. The nucleation of a new recrystallized grain is believed to be due to the growth of an existing deformation-induced subgrain (Holm et al., 2003). At elevated temperatures, a subgrain with a

\footnotetext{
This work was performed at Sandia National Laboratories. Sandia is a multiprogram laboratory operated by Sandia Corporation, a Lockheed Martin Company, for the United States Department of Energy under contract DEAC04-94AL85000.

* Corresponding author.

E-mail address: aabrown@sandia.gov (A.A. Brown).

This article is a U.S. government work, and is not subject to copyright in the United States.
} 
lower level of stored energy will preferentially expand at the expense of neighboring subgrains. The driving force for recrystallization is the difference in energy between the deformed and recrystallized state (Doherty, 2005). If the expanding subgrain reaches a critical size, it becomes a stable recrystallized grain.

Numerous theories exist in the literature for modeling dynamic recrystallization (Sandstrom and Lagneborg, 1975; Busso, 1998; Manonukul and Dunne, 1999; Fan and Yang, 2011). Other models have been presented for static recrystallization, usually based on the work of Johnson and Mehl (1939), Avrami (1939), and Kolmogorov (1937). There are very few models that are capable of modeling both static and dynamic recrystallization (Pietrzyk, 2002; Roucoules et al., 2003; Svyetlichnyy, 2007), and the ones that do exist have recrystallization kinetics or critical recrystallization criteria that depend explicitly on strain or strain rate, thus not being sufficiently general to be able to model transient loading conditions in which the strain rate is not constant.

Among the points of contention in recrystallization literature is what criteria should be used for determining the onset of recrystallization. The two most widely-used methods make use of a critical strain or a critical value of stored energy for initiating recrystallization. Total strain is not a state variable, and thus should not be used to determine the onset of recrystallization. Bailey and Hirsch (1962) used a model in which recrystallization began when a critical value of dislocation density was reached. This is equivalent to a critical stored energy if the stored energy is assumed to be a function of dislocation density only. Others use a critical size for a stable nucleus to determine the onset of recrystallization (Sandstrom and Lagneborg, 1975; Roberts and Ahlblom, 1978). When the subgrain dimension reaches the critical size, recrystallization begins. Wray (1975) based the onset of recrystallization on a critical stored energy. In the current work, no critical criterion was utilized to initiate recrystallization. Rather, the kinetics of recrystallization are modeled based on the mobility of grain and subgrain boundaries under the driving force provided by the stored energy in the dislocation structure. In this manner, it can be used to model multiple-stage loading conditions in which the temperature and strain rate change over the history of the material. The internal state variable theory presented here is capable of modeling both static as well as dynamic recrystallization.

The paper is organized as follows: first, ignoring the effects of recrystallization, we will describe the kinematics and thermodynamics associated with the model. Then the constitutive model will be described without including the effects of recrystallization. Next, the form of the model accounting for recrystallization will be motivated physically. Finally, a comparison between model and experimental results, including static and dynamic recrystallization, will be provided.

\section{Kinematics}

Following the kinematics introduced by Kröner (1960) and further developed by others (Lee and Liu, 1967; Fox, 1968; Lee, 1969), we assume the existence of a purely elastic region that encloses the origin in stress space, and define a multiplicative decomposition of the deformation gradient into elastic and plastic parts. ${ }^{1}$ Similar to Bammann et al. (1996), we further decompose the deformation gradient to explicitly include the effects of thermal expansion:

$$
\boldsymbol{F}=\boldsymbol{F}^{\boldsymbol{e}} \boldsymbol{F}^{\boldsymbol{p}} \boldsymbol{F}^{\theta},
$$

where $\boldsymbol{F}^{e}$ and $\boldsymbol{F}^{p}$ represent the elastic and plastic portions of the deformation, and $\boldsymbol{F}^{\theta}$ represents the portion due to thermal expansion. Locally, the material can theoretically be unloaded from the current configuration, $\mathfrak{B}$, through $\boldsymbol{F}^{e-1}$ to a stress-free intermediate configuration, $\overline{\mathfrak{B}}$, which as noted in Casey and Naghdi (1992), is only uniquely defined up to a rotation due to invariance requirements.

We assume isotropic thermal expansion, given by

$$
\boldsymbol{F}^{\theta}=F^{\theta} \mathbf{I} \approx\left(1+\beta\left(\theta-\theta_{0}\right)\right) \boldsymbol{I},
$$

where $\beta$ is the linear coefficient of thermal expansion and $\theta_{o}$ is the temperature in the reference configuration, $\mathfrak{B}$. The velocity gradient in the current configuration, $\mathfrak{B}$, is defined as

$$
\boldsymbol{L}=\dot{\boldsymbol{F}} \boldsymbol{F}^{-1}=\dot{\boldsymbol{F}}^{e} \boldsymbol{F}^{e-1}+\boldsymbol{F}^{e} \dot{\boldsymbol{F}}^{p} \boldsymbol{F}^{p-1} \boldsymbol{F}^{e-1}+\frac{\dot{F}_{\theta}}{F_{\theta}} \boldsymbol{I}=\boldsymbol{L}^{e}+\boldsymbol{L}^{p}+\boldsymbol{L}^{\theta},
$$

where $\boldsymbol{L}^{e}=\dot{\boldsymbol{F}}^{e} \boldsymbol{F}^{e-1}, \boldsymbol{L}^{p}=\boldsymbol{F}^{e} \dot{\boldsymbol{F}}^{p} \boldsymbol{F}^{p-1} \boldsymbol{F}^{e-1}=\boldsymbol{F}^{e} \overline{\mathbf{L}}^{p} \boldsymbol{F}^{e-1}$, and $\boldsymbol{L}^{\theta}=\frac{\dot{\boldsymbol{F}}_{\theta}}{\bar{F}_{\theta}} \boldsymbol{I}$. We define $\overline{\boldsymbol{L}}^{p}=\dot{\boldsymbol{F}}^{p} \boldsymbol{F}^{p-1}$ as the plastic velocity gradient in the intermediate configuration, and decompose it into symmetric and skew-symmetric parts,

$$
\overline{\boldsymbol{D}}^{p}=\operatorname{sym}\left(\bar{L}^{p}\right) \text { and } \overline{\boldsymbol{W}}^{p}=\operatorname{skew}\left(\bar{L}^{p}\right),
$$

such that ${ }^{2}$

$$
\overline{\mathbf{L}}^{p}=\overline{\boldsymbol{D}}^{p}+\overline{\boldsymbol{W}}^{p} .
$$

The elastic strain in the intermediate configuration is written as

\footnotetext{
${ }^{1}$ See Casey and Naghdi (1992) for a kinematical treatment of the case where the yield surface in stress space does not enclose the origin.

${ }^{2}$ Note that the rate of deformation tensors $\left(\overline{\boldsymbol{D}}, \overline{\boldsymbol{D}}^{p}, \overline{\boldsymbol{D}}^{e}\right)$ and spin tensors $\left(\overline{\boldsymbol{W}}, \overline{\boldsymbol{W}}^{p}, \overline{\boldsymbol{W}}^{e}\right)$ in the intermediate configuration are not the same as would be obtained by pulling back their counterparts from the current configuration. For example, it can be shown that $\boldsymbol{F}^{e T} \boldsymbol{D}^{p} \boldsymbol{F}^{e}=\overline{\boldsymbol{D}}^{p}+\overline{\boldsymbol{L}}^{p T} \boldsymbol{E}^{e}+\boldsymbol{E}^{e} \overline{\boldsymbol{L}}^{p}$ (see Marin et al. (2006)).
} 


$$
\overline{\boldsymbol{E}}^{e}=\frac{1}{2}\left(\boldsymbol{F}^{e T} \boldsymbol{F}^{e}-\boldsymbol{I}\right)=\frac{1}{2}\left(\overline{\boldsymbol{C}}^{e}-\boldsymbol{I}\right),
$$

where $\overline{\boldsymbol{C}}^{e}=\boldsymbol{F}^{e T} \boldsymbol{F}^{e}$. The pullbacks of $\boldsymbol{L}$ and $\boldsymbol{L}^{e}$ to the intermediate configuration are given by

$$
\overline{\boldsymbol{L}}=\boldsymbol{F}^{e-1} \boldsymbol{L} \boldsymbol{F}^{e} \text { and } \quad \overline{\boldsymbol{L}}^{e}=\boldsymbol{F}^{e-1} \dot{\boldsymbol{F}}^{e} .
$$

\section{Thermodynamics}

Following the thermodynamics developed in Coleman and Gurtin (1967), internal state variables are introduced in this section to represent the effects of statistically stored and geometrically necessary dislocations. The Clausius-Duhem inequality in the intermediate configuration takes the form (see Regueiro et al., 2002; Bammann, 2001; Marin et al., 2006):

$$
-\bar{\rho} \dot{\bar{\psi}}-\bar{\rho} \bar{\eta} \dot{\theta}+\left(\overline{\boldsymbol{C}}^{e} \overline{\boldsymbol{S}}\right) \cdot \overline{\mathbf{L}}-\frac{1}{\theta} \overline{\mathbf{Q}} \cdot \bar{\nabla} \theta \geqslant 0 .
$$

where $\bar{\rho}$ is the material density, $\bar{\psi}$ is the Helmholtz free energy, $\bar{\eta}$ is the entropy, $\overline{\boldsymbol{Q}}$ is the heat flux, $\overline{\boldsymbol{S}}$ is the second Piola-Kirchhoff stress pushed forward to the intermediate configuration $\left(\overline{\boldsymbol{S}}=\boldsymbol{F}^{p} \boldsymbol{S F}^{p \mathrm{~T}}\right), \overline{\boldsymbol{C}}^{e} \overline{\boldsymbol{S}}$ is the Mandel stress (see Mandel et al., 1974), and $\bar{\nabla} \theta$ is the gradient of temperature with respect to the intermediate configuration. We assume that the free energy is a function of the elastic strain due to external loads, $\overline{\boldsymbol{E}}^{e}$, the lattice strain due to statistically stored dislocations, $\bar{\varepsilon}_{\mathrm{ssds}}$, the lattice curvature due to geometrically necessary dislocations, ${ }^{3} \bar{\alpha}_{\text {gnds }}$, and the temperature:

$$
\bar{\psi}=\bar{\psi}\left(\overline{\boldsymbol{E}}^{e}, \bar{\varepsilon}_{\mathrm{ssds}}, \bar{\alpha}_{\mathrm{gnds}}, \theta\right) \text {. }
$$

Using the chain rule and noting that $\left(\overline{\boldsymbol{C}}^{e} \overline{\boldsymbol{S}}\right) \cdot \overline{\boldsymbol{L}}=\overline{\boldsymbol{S}} \cdot\left(\overline{\boldsymbol{C}}^{e} \overline{\boldsymbol{L}}^{e}\right)+\left(\overline{\boldsymbol{C}}^{e} \overline{\boldsymbol{S}}\right) \cdot \overline{\boldsymbol{L}}^{p}$ and $\overline{\boldsymbol{C}}^{e} \overline{\boldsymbol{L}}^{e}=F^{e T} \dot{\boldsymbol{F}}^{e}=\dot{\overline{\boldsymbol{E}}}^{e}$, Eq. (8) can then be rewritten as

$$
\left(-\bar{\rho} \frac{\partial \bar{\psi}}{\partial \overline{\boldsymbol{E}}^{e}}+\overline{\boldsymbol{S}}\right) \cdot \dot{\overline{\boldsymbol{E}}}^{e}-\bar{\rho}\left(\frac{\partial \bar{\psi}}{\partial \theta}+\bar{\eta}\right) \dot{\theta}-\bar{\rho} \frac{\partial \bar{\psi}}{\partial \overline{\bar{\varepsilon}}_{\mathrm{ssds}}} \dot{\bar{\varepsilon}}_{\mathrm{ssds}}-\bar{\rho} \frac{\partial \bar{\psi}}{\partial \bar{\alpha}_{\text {gnds }}} \dot{\bar{\alpha}}_{\text {gnds }}+\left(\overline{\boldsymbol{C}}^{e} \overline{\boldsymbol{S}}\right) \cdot \overline{\mathbf{L}}^{p}-\frac{1}{\theta} \overline{\mathbf{Q}} \cdot \bar{\nabla} \theta \geqslant 0 .
$$

Since Eq. (10) must hold for arbitrary elastic strain rates and temperature rates, then by the standard argument (see Coleman and Noll (1963)), it follows that

$$
\overline{\boldsymbol{S}}=\bar{\rho} \frac{\partial \bar{\psi}}{\partial \overline{\boldsymbol{E}}^{e}} \quad \text { and } \quad \bar{\eta}=-\frac{\partial \bar{\psi}}{\partial \theta} .
$$

We then can define stress-like internal variables conjugate to $\bar{\varepsilon}_{\text {ssds }}$ and $\bar{\alpha}_{\text {gnbs }}$ as

$$
\bar{\kappa}=\bar{\rho} \frac{\partial \bar{\psi}}{\partial \bar{\varepsilon}_{\text {ssds }}} \quad \text { and } \quad \bar{\zeta}=\bar{\rho} \frac{\partial \bar{\psi}}{\partial \bar{\alpha}_{\text {gnds }}} .
$$

Eq. (10) then reduces to

$$
\left(\overline{\boldsymbol{C}}^{e} \overline{\boldsymbol{S}}\right) \cdot \overline{\mathbf{L}}^{p}-\bar{\kappa} \dot{\bar{\varepsilon}}_{\text {ssds }}-\bar{\zeta}_{\overline{\bar{\varepsilon}}}^{\text {gnds }}-\frac{1}{\theta} \overline{\mathbf{Q}} \cdot \bar{\nabla} \theta \geqslant 0 .
$$

\section{Constitutive model}

First we will provide the constitutive relations for the free energy, the evolution of the state variables, and the flow rule for plastic deformation, without accounting for recrystallization. The model is a variation of the one proposed in Bammann et al. (1995), to which various modifications have been made over the years, including the nonlocal crystal plasticity model of Bammann (2001) and its phenomenological polycrystalline version (Regueiro et al., 2002).

\subsection{Helmholtz free energy}

The constitutive equations will be presented in the intermediate configuration. Metals in general experience small elastic strains, so we assume the following form for the free energy:

$$
\bar{\rho} \bar{\psi}=\frac{1}{2} \overline{\boldsymbol{E}}^{e} \cdot\left(\lambda(\theta) \overline{\boldsymbol{I}} \otimes \overline{\boldsymbol{I}}+2 \mu(\theta) \overline{\boldsymbol{I}}_{4 \mathrm{th}}\right) \overline{\boldsymbol{E}}^{e}+\frac{1}{2} c_{\bar{\varepsilon}_{\text {ssds }}} \mu(\theta) \bar{\varepsilon}_{\text {ssds }}^{2}+\frac{1}{2} c_{\bar{\alpha}_{\text {gnds }}} \mu(\theta) \bar{\alpha}_{\text {gnds }}^{2}+\bar{g}(\theta),
$$

where $\lambda(\theta)$ and $\mu(\theta)$ are Lamés constants, $\mu(\theta)$ is the shear modulus, $c_{\bar{\varepsilon}_{s d s}}$ and $c_{\bar{\alpha}_{\text {ssds }}}$ are material constants, and $\bar{g}(\theta)$ is the internal thermal energy. Eqs. (11) and (12) then become

$$
\begin{aligned}
& \overline{\boldsymbol{S}}=\lambda(\theta) \operatorname{tr}\left(\overline{\boldsymbol{E}}^{e}\right) \overline{\boldsymbol{I}}+2 \mu(\theta) \overline{\boldsymbol{E}}^{e} \\
& \bar{\kappa}=c_{\bar{\varepsilon}_{\text {ssds }}} \mu(\theta) \bar{\varepsilon}_{\text {ssds }}, \quad \text { and } \bar{\zeta}=c_{\bar{\alpha}_{\text {ssds }}} \mu(\theta) \bar{\alpha}_{\text {gnds }} .
\end{aligned}
$$

\footnotetext{
${ }^{3}$ This is a scalar version of the tensorial curvature introduced in Bammann (2001) and Regueiro et al. (2002).
} 


\subsection{Plastic velocity gradient}

The plastic rate of deformation tensor is related to the equivalent plastic strain rate by

$$
\overline{\boldsymbol{D}}^{p}=\left\|\overline{\boldsymbol{D}}^{p}\right\| \boldsymbol{n}^{p},\left\|\overline{\boldsymbol{D}}^{p}\right\|=\sqrt{\frac{3}{2}} \dot{\bar{\varepsilon}}^{p}, \quad \text { and } \quad \boldsymbol{n}^{p}=\frac{\operatorname{dev} \overline{\mathbf{S}}}{\|\operatorname{dev} \overline{\mathbf{S}}\|} .
$$

where dev $\overline{\mathbf{S}}$ is the deviatoric part of the stress, $\overline{\mathbf{S}}^{4}$ From Frost and Ashby (1982), Regueiro et al. (2002), and Marin et al. (2006), we define the evolution of plastic flow as

$$
\dot{\bar{\varepsilon}}^{p}=f(\theta)\left(\sinh \left[\left\langle\frac{\sqrt{\frac{3}{2}}\|\operatorname{dev} \overline{\boldsymbol{S}}\|}{\bar{\kappa}+Y(\theta)}-1\right\rangle\right]\right)^{n(\theta)},
$$

where $f(\theta), Y(\theta)$, and $n(\theta)$ are scalar functions of temperature. The yield parameter $f(\theta)$ has an Arrhenius-type temperature dependence, $f(\theta)=c_{1} e^{-\frac{c_{2}}{\theta}}$, and $n(\theta)$ and $Y(\theta)$ are defined by $n(\theta)=c_{3}+\frac{c_{4}}{\theta}$ and $Y(\theta)=c_{5} \mu(\theta)$.

In Prantil et al. (1993), the plastic spin, $\overline{\mathbf{W}}^{p}$, is defined in terms of a structure tensor, which is used to describe textureinduced anisotropy. Here, we do not account for texture effects, so we assume $\overline{\mathbf{W}}^{p}=0$.

\subsection{Statistically stored dislocations}

The scalar stress-type variable, $\bar{\kappa}$, can be related to the statistically stored dislocation density by defining the lattice strain due to statistically stored dislocations as (see Taylor, 1934)

$$
\bar{\varepsilon}_{\text {ssds }}=b \sqrt{\bar{\rho}_{\text {ssds }}},
$$

which leads to

$$
\bar{\kappa}=c_{\bar{\varepsilon}_{\text {ssds }}} b \mu(\theta) \sqrt{\bar{\rho}_{\text {ssds }}},
$$

where $b$ is the magnitude of the Burger's vector, and $\bar{\rho}_{\text {ssds }}$ is the density of statistically stored dislocations.

Kocks and Mecking (1979) provided an evolution equation for the statistically stored dislocation density that included terms representing the generation and annihilation of statistically stored dislocations. The generation rate is assumed to be proportional to the rate at which they become immobilized, which is inversely proportional to their mean free path. Dynamic recovery, a thermally activated process, is assumed to be proportional to the dislocation density. A term can also be included to account for static recovery, although no such term is included here. Using the type of hybrid theory conceived of by Estrin and Mecking (1984), we assume the mean free path is influenced by interactions with other dislocations as well as interactions with subgrain boundaries due to geometrically necessary dislocations, which are discussed in the next section. Following Kok et al. (2002), we assume the statistically stored dislocation density evolves with plastic strain according to the following equation:

$$
\dot{\bar{\rho}}_{\text {ssds }}=\left[\frac{k_{1}}{L_{s}}+\frac{k_{2}}{L_{g}}-R_{d}(\theta) \bar{\rho}_{\text {ssds }}\right] \dot{\bar{\varepsilon}}^{p},
$$

where $L_{s}$ and $L_{g}$ are the mean free paths associated with dislocation-dislocation and dislocation-geometrically necessary boundary interactions, $R_{d}(\theta)=c_{6} e^{-c_{7} / \theta}$, and $k_{1}, k_{2}, c_{6}$ and $c_{7}$ are material constants. The mean free path, $L_{s}$, is related to the statistically stored dislocation density by

$$
L_{s} \propto \frac{1}{\sqrt{\bar{\rho}_{\text {ssds }}}} .
$$

As demonstrated in Kok et al. (2002), the term related to $L_{g}$ leads to stage IV hardening behavior. Without its inclusion, the statistically stored dislocation density would approach a steady-state value with continued plastic strain. Instead, as the spacing between geometrically necessary boundaries continues to decrease, the mean free path continues to shrink, leading to stage IV hardening. Following Kok et al. (2002), we define $\bar{\zeta}$ to be a stress-type variable that is proportional to the shear modulus and inversely proportional to the average spacing of the geometrically necessary boundaries, $L_{g}$ :

\footnotetext{
${ }^{4}$ Based on Casey and Naghdi (1992), the following invariance requirements under superposed rigid body motions must be satisfied:

$$
\boldsymbol{F}^{p+}=\overline{\mathbf{Q}} \boldsymbol{F}^{p}, \quad \boldsymbol{F}^{e+}=\boldsymbol{Q} \boldsymbol{F}^{e} \overline{\mathbf{Q}}^{\mathrm{T}},
$$
}

where $\overline{\mathbf{Q}}$ and $\boldsymbol{Q}$ are arbitrary independent proper orthogonal tensors. From these two equations, it follows that

$$
\overline{\boldsymbol{S}}^{+}=\overline{\mathbf{Q} \boldsymbol{S} \overline{\boldsymbol{Q}}^{\mathrm{T}},} \quad \overline{\boldsymbol{E}}^{e+}=\overline{\mathbf{Q} \boldsymbol{E}^{e}} \overline{\mathbf{Q}}^{\mathrm{T}}, \quad \overline{\boldsymbol{D}}^{p+}=\overline{\mathbf{Q} \bar{D}^{p}} \overline{\mathbf{Q}}^{\mathrm{T}}, \quad \text { and } \quad \operatorname{dev} \overline{\boldsymbol{S}}^{+}=\overline{\mathbf{Q}}(\operatorname{dev} \overline{\boldsymbol{S}}) \overline{\mathbf{Q}}^{\mathrm{T}} .
$$

Thus, the constitutive theory is properly invariant. 


$$
\bar{\zeta}=\frac{c_{\bar{\varepsilon}_{\mathrm{sds}}} b \mu(\theta) k_{2}}{k_{1} L_{g}} .
$$

By taking the chain rule of Eq. (19), using Eq. (20), and renaming constants, we get an evolution equation for the hardening variable, $\bar{\kappa}$ :

$$
\dot{\bar{\kappa}}=\frac{\bar{\kappa}}{\mu(\theta)} \frac{d \mu}{d \theta} \dot{\theta}+\left[H(\theta)\left(1+\frac{\bar{\zeta}}{\overline{\bar{\kappa}}}\right)-R_{d}(\theta) \bar{\kappa}\right] \dot{\bar{\varepsilon}}^{p},
$$

where $H(\theta)=c_{8} \mu(\theta)$.

\subsection{Geometrically necessary dislocations}

As a metal is plastically deformed, geometrically necessary dislocations are generated to ensure compatibility for nonhomogeneous materials. In a polycrystalline material, the grains tend to align themselves with the applied loading. Geometrically necessary dislocations facilitate lattice curvature in individual grains, which allows portions of the grains to rotate without leading to cracks, voids, or gaps at the interfaces between grains (see Ashby, 1970). These dislocations can be stored in boundaries, called geometrically necessary boundaries, that separate subgrain regions with misorientation angles existing between crystal lattices on either sides of the boundaries (see Hughes et al., 1997; Hughes and Hansen, 2000). As the deformation progresses, the average misorientation angle across these boundaries increases, and the spacing between decreases.

Bammann (2001) represents geometrically necessary dislocations with a dislocation curvature tensor, defined as the curl of the elastic rotation associated with the polar decomposition of the elastic deformation gradient. Here, as in Kok et al. (2002), we limit ourselves to a scalar misorientation variable, $\bar{\zeta}$, as defined in Eq. (22) to be inversely proportional to the average spacing of the geometrically necessary boundaries. Based on the work of Hughes et al. (1997) and Hughes and Hansen (2000), this implies that $\bar{\zeta}$ is proportional to the average misorientation angle across the boundaries. As the material is deformed, the spacing between the geometrically necessary boundaries decreases, and the misorientation angle across the boundaries increases.

We employ the empirical evolution equation for $L_{g}$, the average spacing between geometrically necessary boundaries, proposed in Kok et al. (2002):

$$
\frac{d}{d \bar{\varepsilon}^{p}}\left(\frac{1}{L_{g}}\right)=c_{L_{g}}\left(\frac{1}{L_{g}}\right)^{1-1 / r},
$$

where $c_{L g}$ and $r$ are parameters, and $\frac{1}{2} \leqslant r \leqslant 1$. The evolution equation for the misorientation variable then follows from Eqs. (22) and (24):

$$
\dot{\bar{\zeta}}=\frac{\bar{\zeta}}{\mu(\theta)} \frac{d \mu}{d \theta} \dot{\theta}+h_{\bar{\zeta}} \mu(\theta)\left(\frac{\bar{\zeta}}{\mu(\theta)}\right)^{1-1 / r}\left|\dot{\bar{\varepsilon}}^{p}\right|,
$$

where $h_{\bar{\zeta}}$ is a constant. The misorientation variable affects the hardening behavior as discussed in the previous section. It also affects the recrystallization kinetics, as discussed in the next section.

\section{Modeling recrystallization}

As mentioned in the introduction, recrystallization nuclei are assumed to be energetically favorable subgrains that grow at the expense of their neighbors. As the material deforms, the average misorientation angle across the geometrically necessary subgrain boundaries increases, which increases the mobility of the boundaries. The migration of the boundaries wipes away the dislocation structure in their path. The driving force for the motion of the geometrically necessary boundaries is the stored energy in the dislocation structure.

\subsection{Recrystallization kinetics}

The evolution of the recrystallization process is tracked through a variable, $X$, representing the volume fraction of recrystallized material. The volume fraction of recrystallized material has a sigmoidal dependence on time. At the beginning of recrystallization $(X \approx 0)$, only a few nuclei exist, so the interfacial area (per volume) between the recrystallized and unrecrystallized regions is small. The interfacial area increases as the existing nuclei grow and new ones are formed, reaches a maximum, and then decreases as the growing nuclei impinge on each other and the amount of unrecrystallized material vanishes ( $X$ approaches the value of 1 ). Speich and Fisher (1966) proposed the empirical relation $A \propto X(1-X)$ for the interfacial area between recrystallized and unrecrystallized regions based on extensive experimental data for nickel. In this work, we assume an empirical relation of the form

$$
A \propto X^{a}(1-X)^{b}
$$

where $a$ and $b$ are constants. 
The evolution rate of the recrystallized volume fraction is given by

$$
\dot{X}=A G,
$$

where $G$ is the average velocity of the interface as it sweeps through the unrecrystallized region (Cahn and Hagel, 1960). ${ }^{5}$ The interface velocity of a geometrically necessary boundary is given by

$$
G=M P,
$$

where $M$ is the mobility and $P$ is the driving force on the boundary (see Gottstein, 2001; Furu et al., 1995; Huang and Humphreys, 1999; Doherty et al., 1997). During recrystallization, we assume the driving force for boundary motion is the stored energy in the unrecrystallized region. Based on the Eq. (14), the driving force takes the form:

$$
P=\frac{1}{2 \mu(\theta)}\left(\frac{\bar{\kappa}^{2}}{c_{\bar{\varepsilon}_{s d s}}}+\frac{\bar{\zeta}^{2}}{c_{\bar{\alpha}_{\text {ssds }}}}\right) \text {. }
$$

Boundary mobility is known to increase with temperature and misorientation (see Doherty et al., 1997; Huang and Humphreys, 2000). We assume the empirical form used by Chen et al. (2002) and Rollett and Holm (1996), but with the misorientation angle replaced by the misorientation variable introduced in Eq. (12):

$$
M=M_{0} e^{-\frac{Q g}{R \theta}}\left(1-e^{-B(\bar{\zeta} / \mu)^{m}}\right) .
$$

where $B, n$, and $M_{o}$ are constants. After making the various substitutions and renaming constants, the recrystallization kinetics described in Eq. (27) become

$$
\dot{X}=R_{\text {rex }}(\theta) Q_{\text {rex }}(\bar{\kappa}, \bar{\zeta}) X^{a}(1-X)^{b},
$$

where $R_{\text {rex }}(\theta)=\frac{1}{\mu} e^{-\frac{c_{\theta}}{\theta}}, Q_{\text {rex }}(\bar{\kappa}, \bar{\zeta})=\left(1-e^{-B(\bar{\zeta} / \mu)^{m}}\right)\left[c_{\bar{\kappa}} \bar{\kappa}^{2}+c_{\bar{\zeta}} \bar{\zeta}^{2}\right]$ and $c_{\theta}, B, n, c_{\bar{\kappa}}$ and $c_{\bar{\zeta}}$ are material constants.

During static recrystallization, one can think of the material as having two-phases: a hard unrecrystallized phase and a soft recrystallized phase. In the unrecrystallized phase, the dislocation density decreases due to static recovery. The recrystallized phase has been wiped clean of dislocations and subgrain boundaries. Dynamic recrystallization is a more complex process. Both the recrystallized and unrecrystallized phases continue to harden (and recover) with deformation, and the material can recrystallize numerous times (Luton and Sellars, 1969; Sandstrom and Lagneborg, 1975; McQueen and Jonas, 1975; Sakai and Jonas, 1984). We introduce the notation $X_{i}$ to represent the volume fraction of material that has undergone at least $i$ cycles of recrystallization. The material can be broken down as follows: unrecrystallized material resides in volume fraction $\left(1-X_{1}\right)$. Material that has recrystallized only once is in volume fraction $\left(X_{1}-X_{2}\right)$, and so on. Each of these regions will have its own set of internal state variables.

The evolution of the first cycle of recrystallization is given by (see Eq. (31)):

$$
\dot{X}_{1}=R_{\text {rex }}(\theta) Q_{\text {rex }}\left(\bar{\kappa}_{1-X_{1}}, \bar{\zeta}_{1-X_{1}}\right) X_{1}^{a}\left(1-X_{1}\right)^{b} .
$$

With continued deformation, the recrystallized region hardens and develops new dislocation structure. The stored energy in the dislocation structure in the region $X_{1}-X_{2}$ provides the driving force for a second cycle of recrystallization. Recalling Eq. (27), the evolution rate of the recrystallized volume fraction $X_{2}$ for the second recrystallization cycle is given by

$$
\dot{X}_{2}=A_{2} G_{2},
$$

where $A_{2}$ is the interfacial area per volume between the fraction of $X_{1}$ undergoing a second cycle of recrystallization, $\frac{X_{2}}{X_{1}}$, and the fraction of $X_{1}$ that has only recrystallized once, $\frac{X_{1}-X_{2}}{X_{1}}$. By analogy to Eq. (26), the interfacial area per volume in $X_{1}$ for the second recrystallization cycle is proportional to $\left(\frac{X_{2}}{X_{1}}\right)^{a}\left(1-\frac{X_{2}}{X_{1}}\right)^{b}$. Hence, the interfacial area per total volume is proportional to $X_{1}\left(\frac{X_{2}}{X_{1}}\right)^{a}\left(1-\frac{X_{2}}{X_{1}}\right)^{b}$. The evolution equation for the recrystallized volume fraction $X_{2}$ is thus

$$
\dot{X}_{2}=R_{\text {rex }}(\theta) Q_{\text {rex }}\left(\bar{\kappa}_{X_{1}-X_{2}}, \bar{\zeta}_{X_{1}-X_{2}}\right) X_{2}\left(\frac{X_{2}}{X_{1}}\right)^{a}\left(1-\frac{X_{2}}{X_{1}}\right)^{b} .
$$

One additional modification is made to the kinetics for the second cycle of recrystallization to correct for the inhomogeneity in the recrystallizing volume fraction $X_{1}-X_{2}$. Consider a real material undergoing multiple cycles of dynamic recrystalliza-

\footnotetext{
${ }^{5}$ In the literature, it is common to assume an Avrami form (see Johnson and Mehl, 1939; Avrami, 1939; Kolmogorov, 1937) for the evolution of the volume fraction of recrystallized material, i.e.

$$
X(t)=1-e^{-a\left(\frac{t}{1 / 2}\right)^{n},}
$$

but this form is based on the assumption of constant velocity, $G$. This is not a valid assumption for dynamic recrystallization, during which the driving force for boundary motion increases with further deformation.
} 
tion. During the second cycle of recrystallization, the material in volume $X_{1}-X_{2}$ has regions that recrystallized during the first cycle much earlier than other regions, and hence have since hardened and developed more dislocation structure. These regions are prone to starting a new wave of recrystallization earlier than regions that just finished recrystallizing the first time and are hence relatively free of dislocation structure. This implies that in the model, Eq. (34) would underpredict the rate of recrystallization, since it uses the homogenized values of the internal state variables in region $X_{1}-X_{2}$. Once the first cycle of recrystallization has completed (i.e. $X_{1} \approx 1$ ), the region $X_{1}-X_{2}$ quickly becomes more homogeneous with continued deformation since no new dislocation-free zones are being added to the region. A correction to account for this behavior is applied to the kinetics of the second recrystallization cycle by way of including a scaling function $f\left(X_{1}\right)$ that decreases to one as $X_{1}$ approaches a value of one:

$$
\begin{aligned}
& \dot{X}_{2}=R_{\text {rex }}(\theta) Q_{\text {rex }}\left(\bar{\kappa}_{X_{1}-X_{2}}, \bar{\zeta}_{X_{1}-X_{2}}\right) X_{1}\left(\frac{X_{2}}{X_{1}}\right)^{a}\left(1-\frac{X_{2}}{X_{1}}\right)^{b} f\left(X_{1}\right), \\
& f\left(X_{1}\right)=1+c\left(1-X_{1}\right) .
\end{aligned}
$$

The same reasoning can be applied for all subsequent cycles of recrystallization, so we write

$$
\dot{X}_{i+1}=R_{r e x}(\theta) Q_{r e x}\left(\bar{\kappa}_{X_{i}-X_{i+1}}, \bar{\zeta}_{X_{i}-X_{i+1}}\right) g\left(X_{i}, X_{i+1}\right),
$$

where $i \geqslant 1$, and we define $g\left(X_{i}, X_{i+1}\right)=X_{i}\left(\frac{X_{i+1}}{X_{i}}\right)^{a}\left(1-\frac{X_{i+1}}{X_{i}}\right)^{b}\left(1+c\left(1-X_{i}\right)\right)$.

\subsection{Evolution of the internal state variables}

Each recrystallized and unrecrystallized volume fraction has its own set of stress-type state variables. The variables in the unrecrystallized phase are assumed to evolve unaltered by the recrystallization process:

$$
\begin{aligned}
& \dot{\bar{\kappa}}_{1-X_{1}}=\frac{\bar{\kappa}_{1-X_{1}}}{\mu(\theta)} \frac{\partial \mu}{\partial \theta} \dot{\theta}+\left[H(\theta)\left(1+\frac{\bar{\zeta}_{1-X_{1}}}{\bar{\kappa}_{1-X_{1}}}\right)-R_{d}(\theta) \bar{\kappa}_{1-X_{1}}\right] \dot{\bar{\varepsilon}}^{p}, \\
& \dot{\bar{\zeta}}_{1-X_{1}}=\frac{\bar{\zeta}_{1-X_{1}}}{\mu(\theta)} \frac{\partial \mu}{\partial \theta} \dot{\theta}+h_{\bar{\zeta}} d^{-1} \bar{\zeta}_{1-X_{1}}^{1-1 / r} \dot{\bar{\varepsilon}}^{p} .
\end{aligned}
$$

The evolution of the variables in the recrystallized phases is derived below.

For the region that has recrystallized once, $X_{1}-X_{2}$, the volume fraction will increase by $d X_{1}$ and decrease by $d X_{2}$ during the time increment, $d t$, as the first and second recrystallization cycles progress. ${ }^{6}$ At time $t+d t$, the portion $d X_{1}$ will have a value of zero for the isotropic hardening variable since we assume that recrystallization removes the dislocation structure. The region $X_{1}(t)-X_{2}(t)-d X_{2}$ will have hardened and/or recovered during the time increment $d t$ in the same manner as it would if no recrystallization had been occurring, so the isotropic hardening variable at time $t+d t$ is given by

$$
\bar{\kappa}_{X_{1}(t)-X_{2}(t)-d X_{2}}(t+d t) \approx \bar{\kappa}_{X_{1}(t)-X_{2}(t)}(t)+\left(\frac{\bar{\kappa}_{X_{1}-X_{2}}}{\mu(\theta)} \frac{\partial \mu}{\partial \theta} \dot{\theta}+\left[H(\theta)\left(1+\frac{\bar{\zeta}_{X_{1}-X_{2}}}{\bar{\kappa}_{X_{1}-X_{2}}}\right)-R_{d}(\theta) \bar{\kappa}_{X_{1}-X_{2}}\right]\left|\dot{\varepsilon}^{p}\right|\right) d t .
$$

The isotropic hardening variable in the region $X_{1}-X_{2}$ at time $t+d t$ is found using a rule of mixtures:

$$
\bar{\kappa}_{X_{1}(t+d t)-X_{2}(t+d t)}(t+d t)=\frac{\bar{\kappa}_{X_{1}(t)-X_{2}(t)-d X_{2}}(t+d t)\left(X_{1}(t)-X_{2}(t)-d X_{2}\right)+0 d X_{1}}{X_{1}(t)-X_{2}(t)+d X_{1}-d X_{2}} .
$$

Combining Eqs. (38) and (39) and taking the limit as $d t \rightarrow 0$, we get

$$
\dot{\bar{\kappa}}_{X_{1}-X_{2}}=\frac{\bar{\kappa}_{X_{1}-X_{2}}}{\mu(\theta)} \frac{\partial \mu}{\partial \theta} \dot{\theta}+\left[H(\theta)\left(1+\frac{\bar{\zeta}_{X_{1}-X_{2}}}{\bar{\kappa}_{X_{1}-X_{2}}}\right)-R_{d}(\theta) \bar{\kappa}_{X_{1}-X_{2}}\right]\left|\dot{\varepsilon}^{p}\right|-\bar{\kappa}_{X_{1}-X_{2}} \frac{\dot{X}_{1}}{X_{1}-X_{2}} .
$$

Similar reasoning is used to add an additional softening term to the evolution equation for the misorientation variable in the recrystallized volume. The evolution equations for the internal state variables in the region $X_{i}-X_{i+1}$ take the form

$$
\begin{aligned}
& \dot{\bar{\kappa}}_{X_{i}-X_{i+1}}=\frac{\bar{\kappa}_{X_{i}-X_{i+1}}}{\mu(\theta)} \frac{\partial \mu}{\partial \theta} \dot{\theta}+\left[H(\theta)\left(1+\frac{\bar{\zeta}_{X_{1}-X_{2}}}{\bar{\kappa}_{X_{1}-X_{2}}}\right)-R_{d}(\theta) \bar{\kappa}_{X_{1}-X_{2}}\right]\left|\dot{\varepsilon}^{p}\right|-\bar{\kappa}_{X_{i}-X_{i+1}} \frac{\dot{X}_{i}}{X_{i}-X_{i+1}}, \\
& \dot{\bar{\zeta}}_{X_{i}-X_{i+1}}=\frac{\bar{\zeta}_{X_{i}-X_{i+1}}}{\mu(\theta)} \frac{\partial \mu}{\partial \theta} \dot{\theta}+h_{\bar{\zeta}} \bar{\zeta}_{X_{i}-X_{i+1}}^{1-r}\left|\dot{\varepsilon}^{p}\right|-\bar{\zeta}_{X_{i}-X_{i+1}} \frac{\dot{X}_{i}}{X_{i}-X_{i+1}}
\end{aligned}
$$

for $i \geqslant 1$. We assume that each region experiences the same plastic strain rate.

\footnotetext{
${ }^{6}$ During dynamic recrystallization, multiple cycles of recrystallization can occur simultaneously (Luton and Sellars, 1969).
} 


\subsection{Model in iterative form}

The evolution equations for the variables in each region and the rates of recrystallization for each cycle can be written in a more concise form if we recall that $X_{i}$ is defined as the volume fraction of material that has undergone at least $i$ cycles of recrystallization and note that $X_{0}=1$ and $\dot{X}_{0}=0$ :

$$
\begin{aligned}
& \dot{X}_{i+1}=R_{\text {rex }}(\theta) Q_{\text {rex }}\left(\bar{\kappa}_{X_{i}-X_{i+1}}, \bar{\zeta}_{X_{i}-X_{i+1}}\right) g\left(X_{i}, X_{i+1}\right), \\
& \dot{\bar{\kappa}}_{X_{i}-X_{i+1}}=\frac{\bar{\kappa}_{X_{i}-X_{i+1}}}{\mu(\theta)} \frac{\partial \mu}{\partial \theta} \dot{\theta}+\left[H(\theta)\left(1+\frac{\bar{\zeta}_{X_{i}-X_{i+1}}}{\bar{\kappa}_{X_{i}-X_{i+1}}}\right)-R_{d}(\theta) \bar{\kappa}_{X_{i}-X_{i+1}}\right]\left|\dot{\varepsilon}^{p}\right|-\bar{\kappa}_{X_{i}-X_{i+1}} \frac{\dot{X}_{i}}{X_{i}-X_{i+1}}, \\
& \dot{\bar{\zeta}}_{X_{i}-X_{i+1}}=\frac{\bar{\zeta}_{X_{i}-X_{i+1}}}{\mu(\theta)} \frac{\partial \mu}{\partial \theta} \dot{\theta}+h_{\bar{\zeta}} \mu(\theta)\left(\frac{\bar{\zeta}_{X_{i}-X_{i+1}}}{\mu(\theta)}\right)^{1-1 / r}\left|\dot{\varepsilon}^{p}\right|-\bar{\zeta}_{X_{i}-X_{i+1}} \frac{\dot{X}_{i}}{X_{i}-X_{i+1}}
\end{aligned}
$$

for $i \geqslant 0$.

For a material that is undergoing multiple (say, $n$ ) recrystallization cycles, the average value for the isotropic hardening variable used in the flow rule is calculated using a rule of mixtures:

$$
\bar{\kappa}=\sum_{i=0}^{n-1} \bar{\kappa}_{X_{i}-X_{i+1}}\left(X_{i}-X_{i+1}\right)+\bar{\kappa}_{X_{n}} X_{n}
$$

To avoid singularities at time $t=0$, small initial values were assigned to the isotropic hardening variables, misorientation variables, and volume fractions: $\kappa_{i}=10^{-6}, \zeta_{i}=10^{-6}$ and $X_{i}=10^{-6} X_{i+1}$ for $i \geqslant 1$. A recursive algorithm is employed in which the values of the variables are cycled when $X_{1}$ gets sufficiently close to one. In the simulations shown in the next section, five cycles of recrystallization were tracked (i.e. $n=5$ ), and when $X_{1}$ became greater than 0.999 , the variable values were replaced as follows:

$$
\begin{array}{llll}
X_{i}^{\text {new }}=X_{i+1}^{\text {old }}, & \bar{\kappa}_{X_{i-1}-X_{i}}^{\text {new }}=\bar{\kappa}_{X_{i}-X_{i+1}}^{\text {old }}, & \bar{\zeta}_{X_{i-1}-X_{i}}^{\text {new }}=\bar{\zeta}_{X_{i}-X_{i+1}}^{\text {old }}, & (i=1,2,3,4) . \\
X_{n}^{\text {new }}=10^{-6} X_{n}^{\text {old }}, & \bar{\kappa}_{X_{n}}^{\text {new }}=0, & \bar{\zeta}_{X_{n}}^{\text {new }}=10^{-6} \bar{\zeta}_{X_{n}}^{\text {old }},
\end{array}
$$

\section{Comparison to experiments}

To see how the model performed against experimental data, we reduced the model for the case of uniaxial stress at constant temperature, and implemented the one-dimensional version in the parameter-fitting program, Bfit (Lathrop, 1997). The implementation utilized the recursive algorithm presented in the last section for $n=5$. For uniaxial stress, let $\sigma$ represent the only non-vanishing component of the Cauchy stress tensor and $\varepsilon$ represent the axial component of the Eulerian strain tensor. After making approximations for small elastic strains, it can be shown that the model reduces to the following set of equations, written here in the current configuration:

$$
\begin{aligned}
& \dot{\sigma}=E\left(\dot{\varepsilon}-\dot{\varepsilon}^{p}\right) \\
& \dot{\varepsilon}^{p}=f(\theta)\left(\sinh \left[\left\langle\frac{\sigma}{\kappa+Y(\theta)}-1\right\rangle\right]\right)^{n(\theta)}, \\
& \kappa=\sum_{i=0}^{n-1} \kappa_{X_{i}-X_{i+1}}\left(X_{i}-X_{i+1}\right)+\kappa_{X_{n}} X_{n}, \\
& \dot{\kappa}_{X_{i}-X_{i+1}}=\left[H(\theta)\left(1+\frac{\zeta_{X_{i}-X_{i+1}}}{\kappa_{X_{i}-X_{i+1}}}\right)-R_{d}(\theta) \kappa_{X_{i}-X_{i+1}}\right]\left|\dot{\varepsilon}^{p}\right|-\kappa_{X_{i}-X_{i+1}} \frac{\dot{X}_{i}}{X_{i}-X_{i+1}}, \\
& \dot{\zeta}_{X_{i}-X_{i+1}}=h_{\zeta} \mu(\theta)\left(\frac{\zeta_{X_{i}-X_{i+1}}}{\mu(\theta)}\right)^{1-1 / r}\left|\dot{\varepsilon}^{p}\right|-\zeta_{X_{i}-X_{i+1}} \frac{\dot{X}_{i}}{X_{i}-X_{i+1}}, \\
& \dot{X}_{i+1}=R_{\text {rex }}(\theta) Q_{\text {rex }}\left(\kappa_{X_{i}-X_{i+1}}, \zeta_{X_{i}-X_{i+1}}\right) g\left(X_{i}, X_{i+1}\right) .
\end{aligned}
$$

Using Bfit, the material parameters were optimized to experimental data from Tanner and McDowell (1999) for OFHC copper. The experiments were conducted over a wide range of temperatures $\left(25-541{ }^{\circ} \mathrm{C}\right)$ and strain rates $(0.0004-5200 / \mathrm{s})$, and exhibited both single and multiple-peak recrystallization behavior. Based on copper data in Ledbetter and Naimon (1974), the shear modulus was assumed to vary linearly with temperature in the range of interest according to $\mu$ $(\theta)=5.47 \mathrm{e} 4-34.1 \theta \mathrm{MPa}$. Using this temperature-dependent shear modulus, the other parameters were optimized to the compression data using Bfit. The results of the optimization are presented in Fig. 1 . The constitutive function $n(\theta)$ turned out to be a constant, and the value of $c_{\bar{\zeta}}$ was zero. The values of all non-zero parameters are listed in Table 1.

The model captures the material response quite well over the full range of temperatures and strain rates, including the single and multiple-peak behavior. For example, at a strain rate of $0.0004 / \mathrm{s}$, three types of behavior are observed: at low temperatures, no dynamic recrystallization is apparent in the stress response; at intermediate temperatures, single-peak 

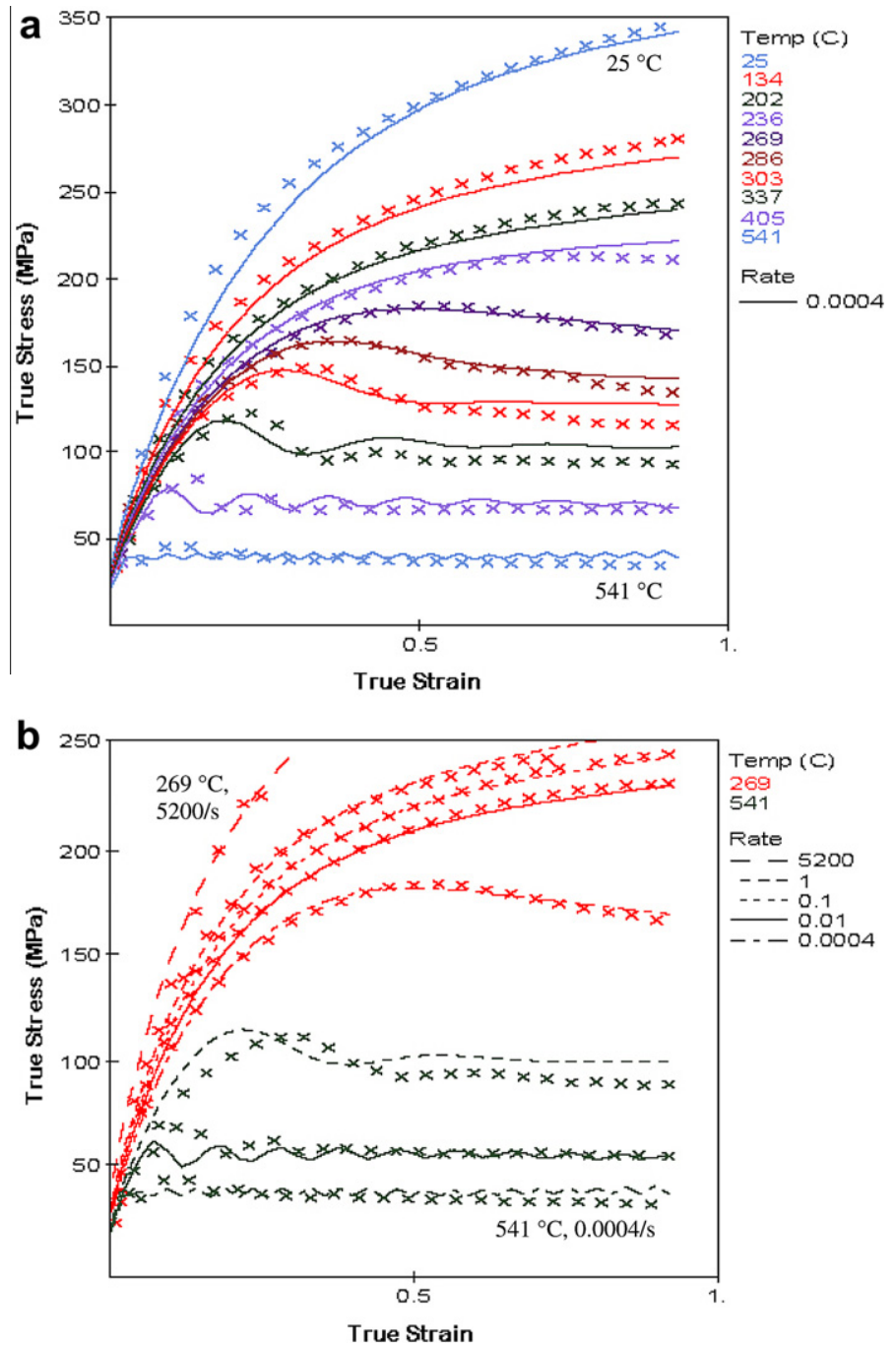

Fig. 1. Comparison between model (lines) and experimental data (symbols) from Tanner and McDowell (1999) for OFHC copper over a range of (a) temperatures and (b) strain rates. The model captures both single and multiple peak behavior.

Table 1

The complete list of non-zero parameters resulting from the optimization based on experimental data from Tanner and McDowell (1999) for OFHC copper.

\begin{tabular}{llll}
\hline Function & Parameter & Value & Units \\
\hline$f(\theta)$ & $c_{1}$ & $2.14 \mathrm{E}-01$ & $1 / \mathrm{s}$ \\
$n$ & $c_{2}$ & $5.22 \mathrm{E}+00$ & $\mathrm{~K}$ \\
$Y(\theta)$ & $c_{3}$ & $1.64 \mathrm{E}+01$ & - \\
$H(\theta)$ & $c_{5}$ & $4.64 \mathrm{E}-04$ & - \\
$R_{d}(\theta)$ & $c_{8}$ & $1.37 \mathrm{E}-02$ & - \\
& $c_{6}$ & $8.80 \mathrm{E}+00$ & $\mathrm{~K}$ \\
$h(\zeta)$ & $c_{7}$ & $2.41 \mathrm{E}+02$ & $1 / \mathrm{s}$ \\
& $h_{\zeta}$ & $2.17 \mathrm{E}-05$ & - \\
$R_{\text {rex }}(\theta)$ & $r$ & $6.57 \mathrm{E}-01$ & $\mathrm{~K}$ \\
$Q_{\text {rex }}(\kappa, \zeta)$ & $c_{\theta}$ & $1.80 \mathrm{E}+04$ & - \\
& $B$ & $1.50 \mathrm{E}+09$ & - \\
$g\left(X_{i}, X_{i+1}\right)$ & $m$ & $2.88 \mathrm{E}+00$ & $\mathrm{~K} /(\mathrm{MPa} \mathrm{s})$ \\
& $c_{\kappa}$ & $8.70 \mathrm{E}+11$ & - \\
& $a$ & $2.25 \mathrm{E}-01$ & - \\
\hline
\end{tabular}


recrystallization is observed; and at high temperatures, multiple-peak recrystallization occurs. These trends are evident in both the model and the experimental results in Fig. 1(a).

Whereas Fig. 1(a) focuses on the temperature dependence, the strain-rate dependence is shown in Fig. 1 (b). At $269{ }^{\circ} \mathrm{C}$, single-peak recrystallization occurs at $0.0004 / \mathrm{s}$, but no recrystallization softening occurs during loading at rates between $0.01 / \mathrm{s}$ through $5200 / \mathrm{s}$. At $541{ }^{\circ} \mathrm{C}$, multiple-peak recrystallization occurs at rates $0.0004 / \mathrm{s}$ through $1 / \mathrm{s}$. This behavior is seen in both the model and the experimental response.

The single-peak behavior seen at $0.0004 / \mathrm{s}$ at temperatures between 269 and $303{ }^{\circ} \mathrm{C}$ is a result of multiple cycles of recrystallization occurring concurrently. The evolution of the state variables is elucidated in Figs. $2-5$ for loading at $303^{\circ} \mathrm{C}$. The shape of the stress-strain curve is primarily determined by the evolution of the isotropic hardening variable, shown in Fig. 2. The value of the isotropic hardening variable is the sum of the contributions from the various volume fractions, according to Eq. $(46)_{3}$. The kinetics of the recrystallization cycles are shown in Fig. 3. Each curve has the typical sigmoidal shape expected during recrystallization. The isotropic hardening and misorientation variables for each volume fraction are shown in Figs. 4 and 5, respectively.

Initially, all of the strength is based on the unrecrystallized volume fraction, $1-X_{1}$. In Fig. 2 , this contribution is seen to initially increase as the unrecrystallized volume fraction hardens, but it decreases as the first cycle of recrystallization occurs.

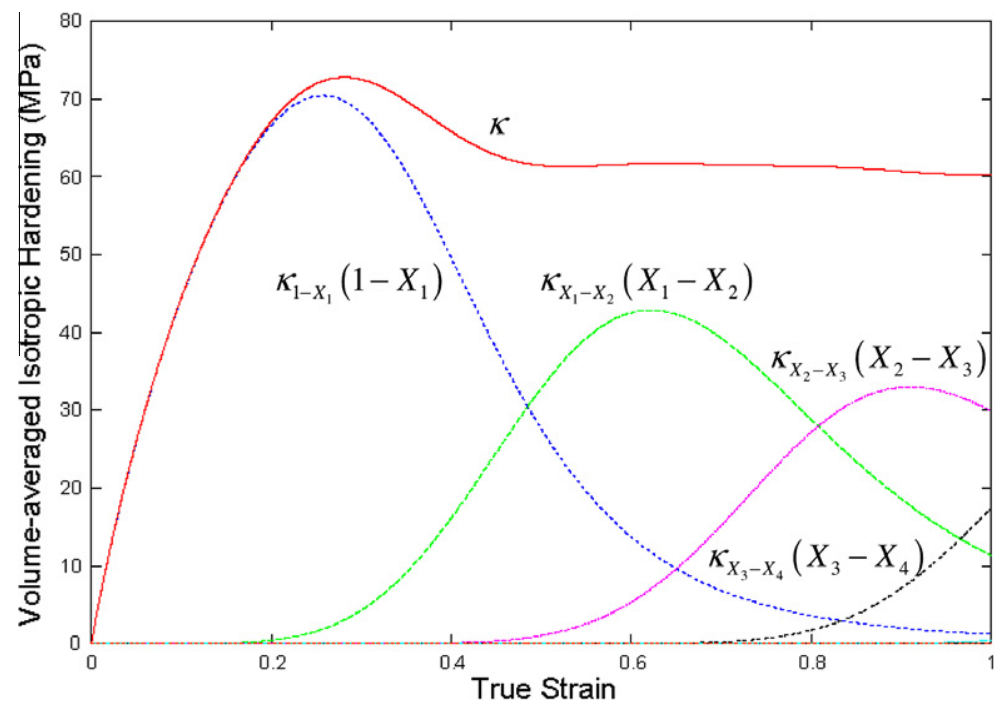

Fig. 2. The contributions to the average isotropic hardening variable from each volume fraction for a strain rate of $0.0004 / \mathrm{s}$ at $303{ }^{\circ} \mathrm{C}$.

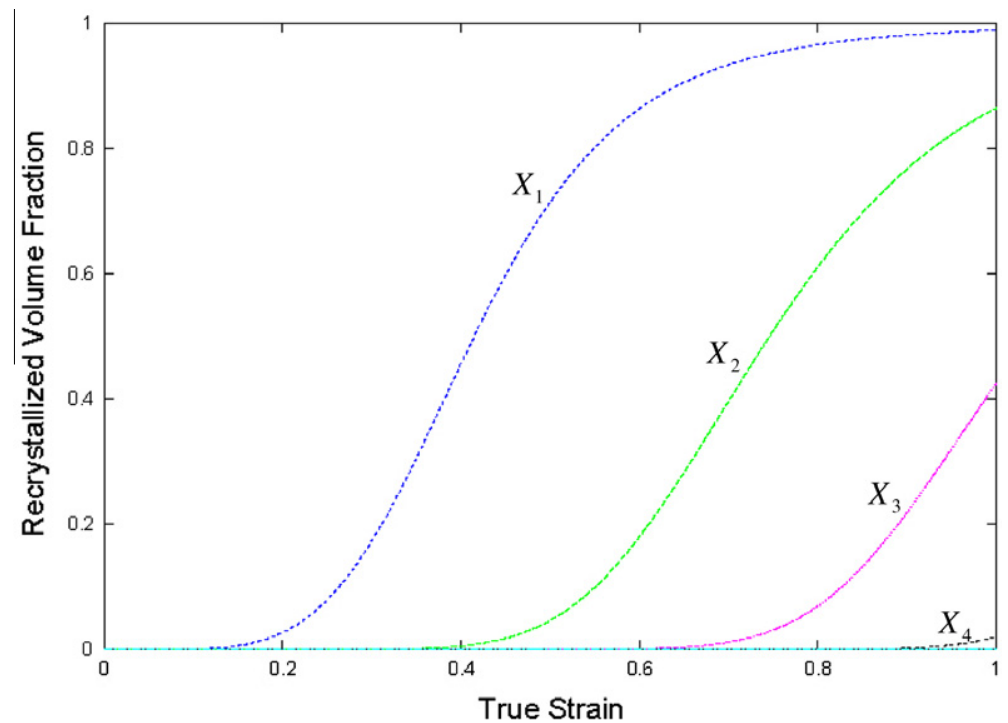

Fig. 3. The evolution of the recrystallization cycles for a strain rate of $303^{\circ} \mathrm{C}$ at $0.0004 / \mathrm{s}$. 


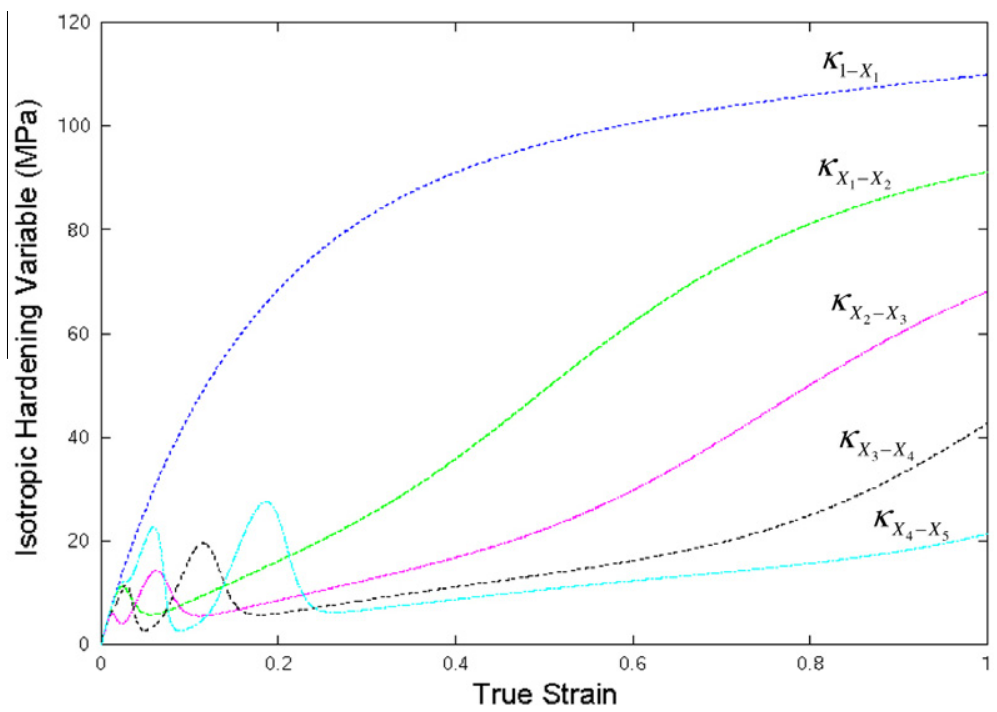

Fig. 4. The isotropic hardening variable in each volume fraction for a strain rate of $303^{\circ} \mathrm{C}$ at $0.0004 / \mathrm{s}$.

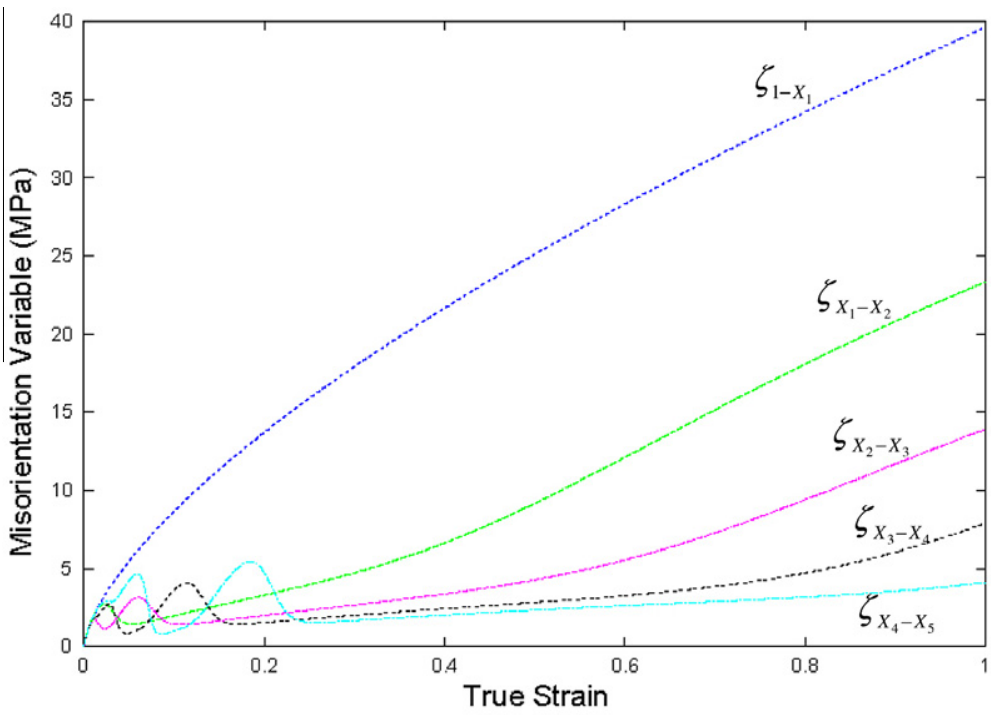

Fig. 5. The misorientation variable in each volume fraction for a strain rate of $0.0004 / \mathrm{s}$ at $303^{\circ} \mathrm{C}$.

The peak value of the isotropic hardening variable (and thus the peak stress) occur after sufficient recrystallization has occurred to make the contribution of the recrystallized volume fraction, $X_{1}-X_{2}$, non-negligible. The contribution of the volume fraction of material that has recrystallized only once rises as $X_{1}-X_{2}$ increases and as the isotropic hardening variable in that volume fraction increases. It then falls as the second cycle of recrystallization occurs. Similar trends are seen for the contributions to the average isotropic hardening variable from the volume fractions of material that have recrystallized twice and three times. In Fig. 2, it is seen that at higher strains, up to four volume fractions have finite contributions to the strength of the material.

The isotropic hardening variable in the unrecrystallized volume fraction, $\kappa_{1-X_{1}}$, is seen to increase monotonically with strain, although its rate decreases as dynamic recovery increases. The misorientation variable, $\zeta_{1-X_{1}}$, increases as the subgrain spacing decreases due to increasing strain. Like in Kok et al. (2002), it is the monotonic increase in the misorientation variable, $\zeta_{1-X_{1}}$, that leads to the stage IV hardening seen in the isotropic hardening variable, $\kappa_{1-X_{1}}$, in the unrecrystallized volume fraction. If the model did not include the misorientation variable, $\kappa_{1-X_{1}}$ would reach a steady-state value where hardening and recovery balanced each other. With the misorientation variable, the decreasing subgrain spacing decreases the mean free path of statistically stored dislocations, thus increasing the hardening rate. As the isotropic hardening and 
misorientation variables increase, the driving force for recrystallization and the boundary mobility also increase, leading to the first cycle of recrystallization.

The isotropic hardening variable in the volume fraction that has recrystallized only once, $\kappa_{X_{1}-X_{2}}$, increases initially due to hardening, then decreases as newly recrystallized material is added to that volume fraction. As the volume fraction $X_{1}-X_{2}$ increases, the influence of hardening dominates over the softening due to newly recrystallized material. The misorientation variable, $\zeta_{X_{1}-X_{2}}$, increases as strain reduces the subgrain spacing, but as newly recrystallized material is added to the volume fraction $X_{1}-X_{2}$, the average subgrain spacing in $X_{1}-X_{2}$ can actually increase, causing a reduction in $\zeta_{X_{1}-X_{2}}$. Subsequent straining will eventually dominate over the addition of newly recrystallized material, causing a monotonic increase in $\zeta_{X_{1}-X_{2}}$ at higher strains. After sufficient hardening, the driving force and boundary mobility are high enough to induce a second recrystallization cycle. Similar trends are seen in the state variables for the volume fractions of material that have recrystallized twice, three times, etc.

Whether dynamic recrystallization exhibits single or multiple-peak behavior depends on how much overlap there is between various cycles of recrystallization (see Luton and Sellars, 1969). This can be seen by comparing the response at $303{ }^{\circ} \mathrm{C}$ in Figs. 2-5 to that seen at $337^{\circ} \mathrm{C}$ in Figs. $6-8$. At $337^{\circ} \mathrm{C}$, the recrystallization cycles occur over smaller strain intervals and

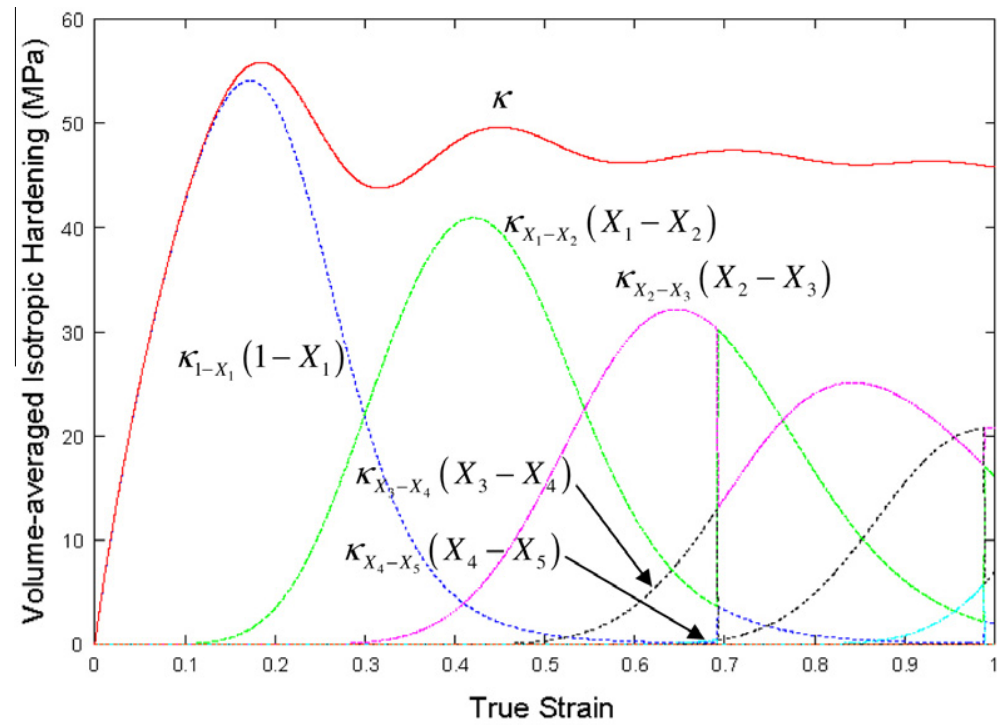

Fig. 6. The contributions to the average isotropic hardening variable from each volume fraction for a strain rate of $0.0004 / \mathrm{s}$ at $337{ }^{\circ} \mathrm{C}$.

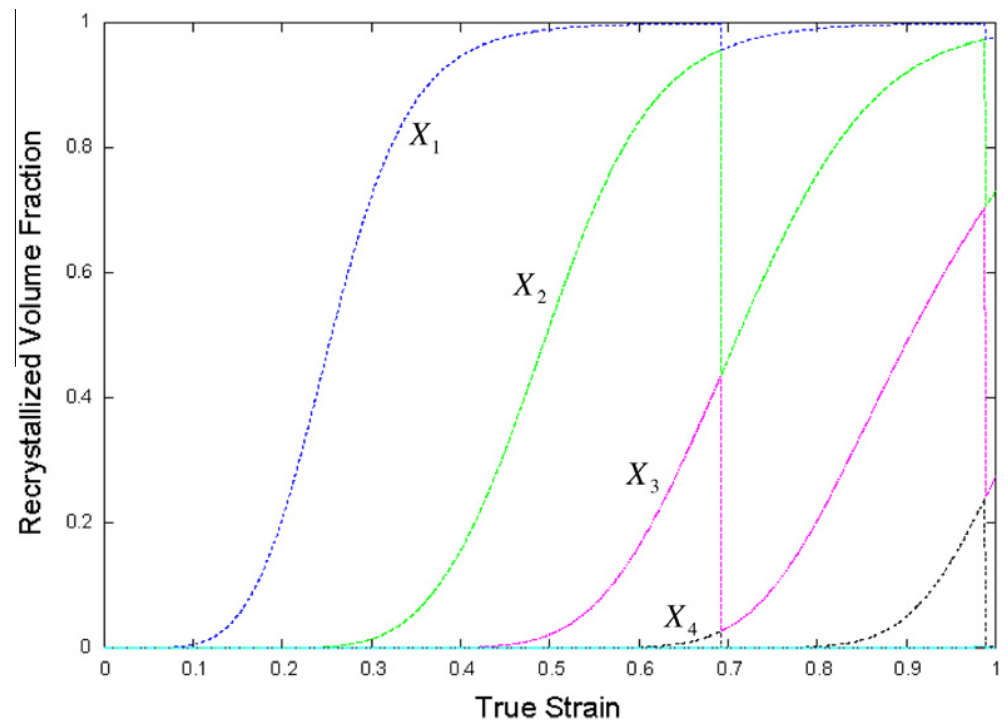

Fig. 7. The evolution of the recrystallization cycles for a strain rate of $337^{\circ} \mathrm{C}$ at $0.0004 / \mathrm{s}$. 


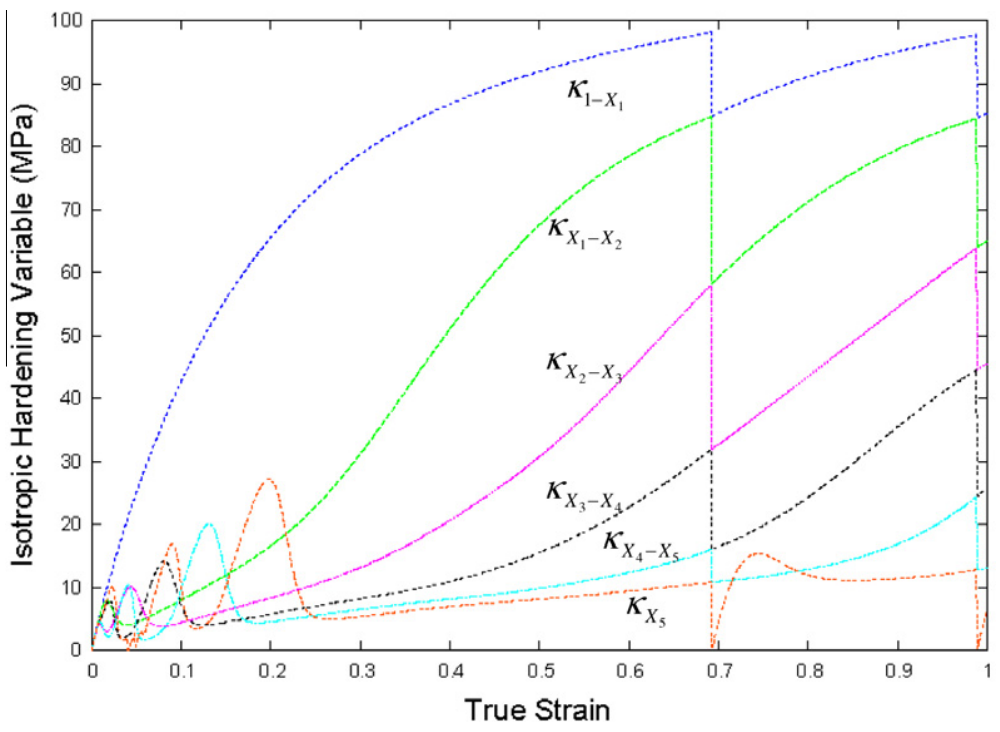

Fig. 8. The isotropic hardening variable in each volume fraction for a strain rate of $337^{\circ} \mathrm{C}$ at $0.0004 / \mathrm{s}$.

overlap one another less than at $303^{\circ} \mathrm{C}$. The actual peak and valley behavior is clarified by revisiting Eq. (46) $)_{3}$; a stress peak or valley will occur when the rate of hardening equals zero, i.e. when

$$
\dot{\kappa}=\sum_{i=0}^{n-1} \frac{d}{d t}\left[\kappa_{X_{i}-X_{i+1}}\left(X_{i}-X_{i+1}\right)\right]+\frac{d}{d t}\left[\kappa_{X_{n}} X_{n}\right]=0,
$$

where the rate of each bracketed quantity represents the rate of change of the contribution to the isotropic hardening variable of a given volume fraction. For example, the rate of change of the contribution of the volume fraction of material that has recrystallized only once, $X_{1}-X_{2}$ will be positive or negative depending on the balance between the rate of hardening and recovery in the volume fraction, given by $\dot{\kappa}_{X_{1}-X_{2}}$, and the rate of change in the volume fraction itself, $\dot{X}_{1}-\dot{X}_{2}$ :

$$
\frac{d}{d t}\left[\kappa_{X_{1}-X_{2}}\left(X_{1}-X_{2}\right)\right]=\dot{\kappa}_{X_{1}-X_{2}}\left(X_{1}-X_{2}\right)+\kappa_{X_{1}-X_{2}}\left(\dot{X}_{1}-\dot{X}_{2}\right) .
$$

Figs. 9-11 show the evolution of the individual terms in Eq. (47) for the temperatures $269^{\circ} \mathrm{C}, 303^{\circ} \mathrm{C}$, and $337^{\circ} \mathrm{C}$, respectively. At $269^{\circ} \mathrm{C}$, multiple cycles of recrystallization are overlapping, and each volume fraction contributes to the average isotropic hardening variable over large strain intervals. Although the rate of hardening is initially positive, it drops to an

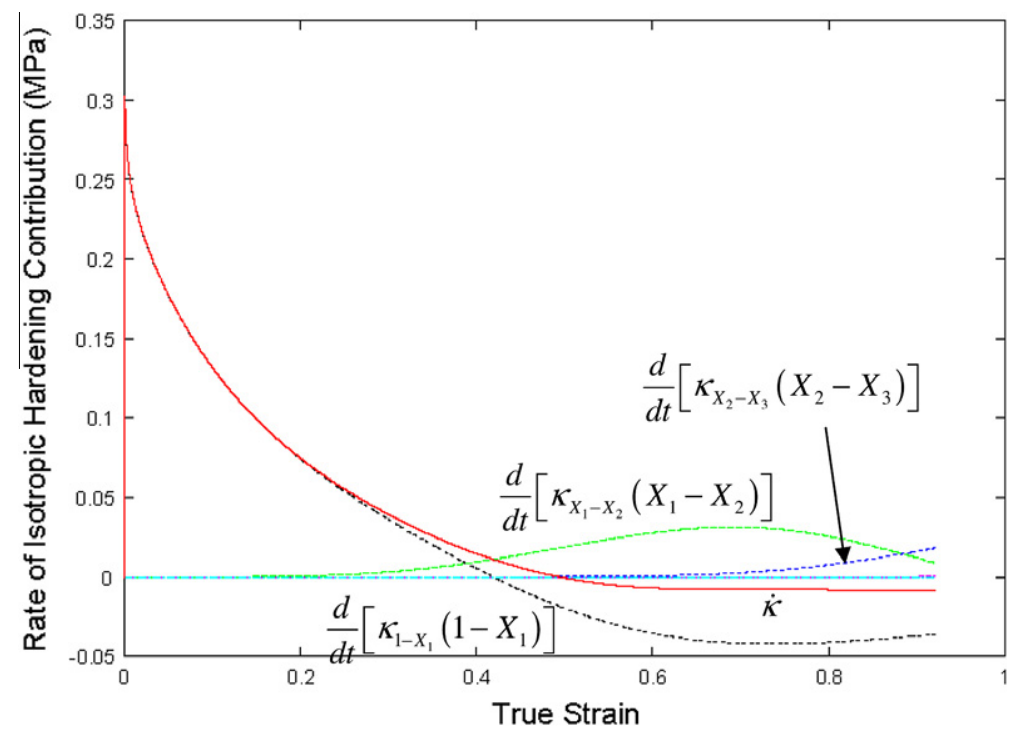

Fig. 9. The rates of change in the contributions to the average isotropic hardening variable from each volume fraction for a strain rate of $269{ }^{\circ} \mathrm{C}$ at $0.0004 / \mathrm{s}$. 


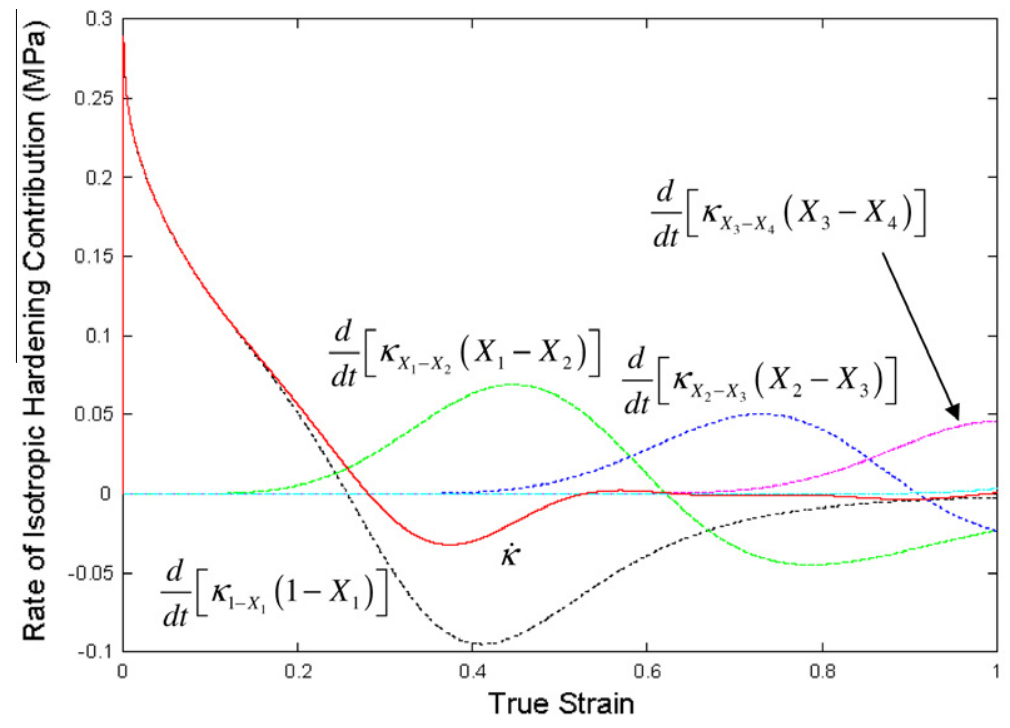

Fig. 10. The rates of change in the contributions to the average isotropic hardening variable from each volume fraction for a strain rate of $0.0004 / \mathrm{s}$ at $303^{\circ} \mathrm{C}$.

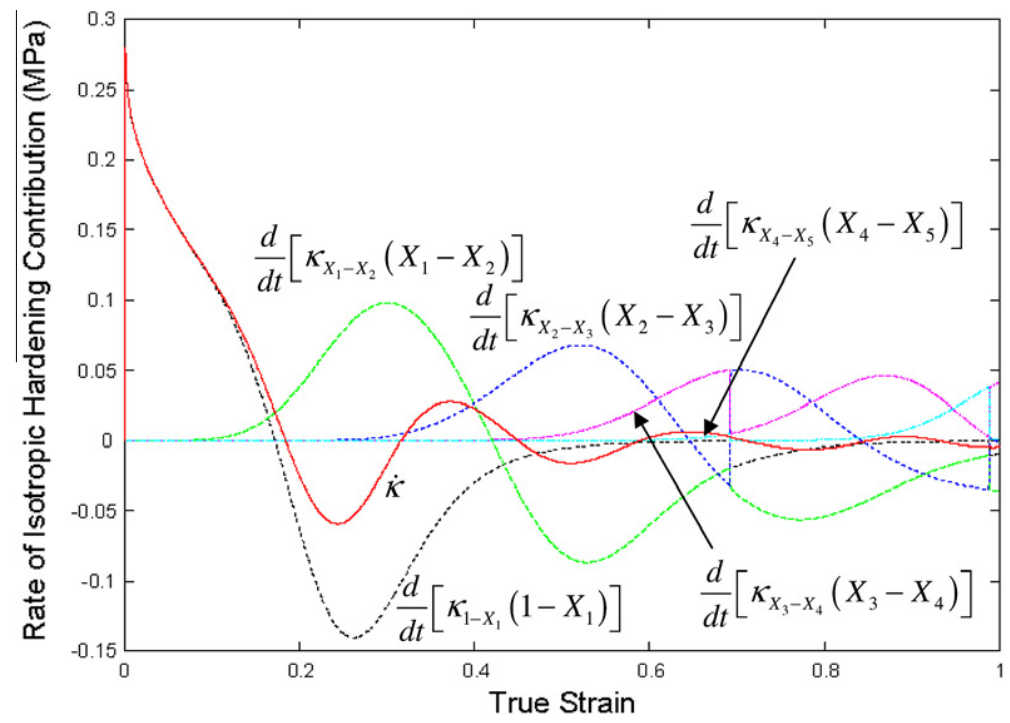

Fig. 11. The rates of change in the contributions to the average isotropic hardening variable from each volume fraction for a strain rate of $0.0004 / \mathrm{s}$ at $337{ }^{\circ} \mathrm{C}$.

almost constant negative value at larger strains. This leads to the single-peak behavior seen at $0.0004 / \mathrm{s}$ for $269{ }^{\circ} \mathrm{C}$ in Fig. 1 (a). At $303{ }^{\circ} \mathrm{C}$, multiple cycles of recrystallization are overlapping, but each volume fraction impacts the overall response over a smaller strain interval. The rate of hardening is initially positive, then drops to negative value, and finally appears to settle at zero for larger strains. The result is once again single-peak behavior. For $337^{\circ} \mathrm{C}$, the initial recrystallization cycle is nearing completion before much of the second cycle has had a chance to occur. At increasing strains, the subsequent cycles overlap more and more, leading to multiple-peak recrystallization with diminishing amplitudes for the peaks and valleys.

In Figs. 6-8, and 11, the iteration scheme in Eq. (45) occurs when the first recrystallization cycle reaches 0.999 . The plots show that the error induced in the response due to the iteration is negligible.

Validation of the model was performed using the parameters that were optimized to single-stage compression to predict multi-stage loading in which specimens are compressed at $0.0004 / \mathrm{s}$ at $269{ }^{\circ} \mathrm{C}$ to strain levels of 0.3 and 0.5 , held for times ranging from $30 \mathrm{~s}$ to $600 \mathrm{~s}$, and then further compressed at 0.0004/s (see Fig. 12). For longer hold times, static recrystallization occurs and reduces the material strength, so that the yield stress upon reloading is reduced. When the first loading stage is stopped at a strain of 0.3 , the driving force and mobility are low, leading to a low rate of static recrystallization and thus only a small decrease in the yield stress, even for a hold time of five minutes (Fig. 12(a)). During the second loading stage, the 

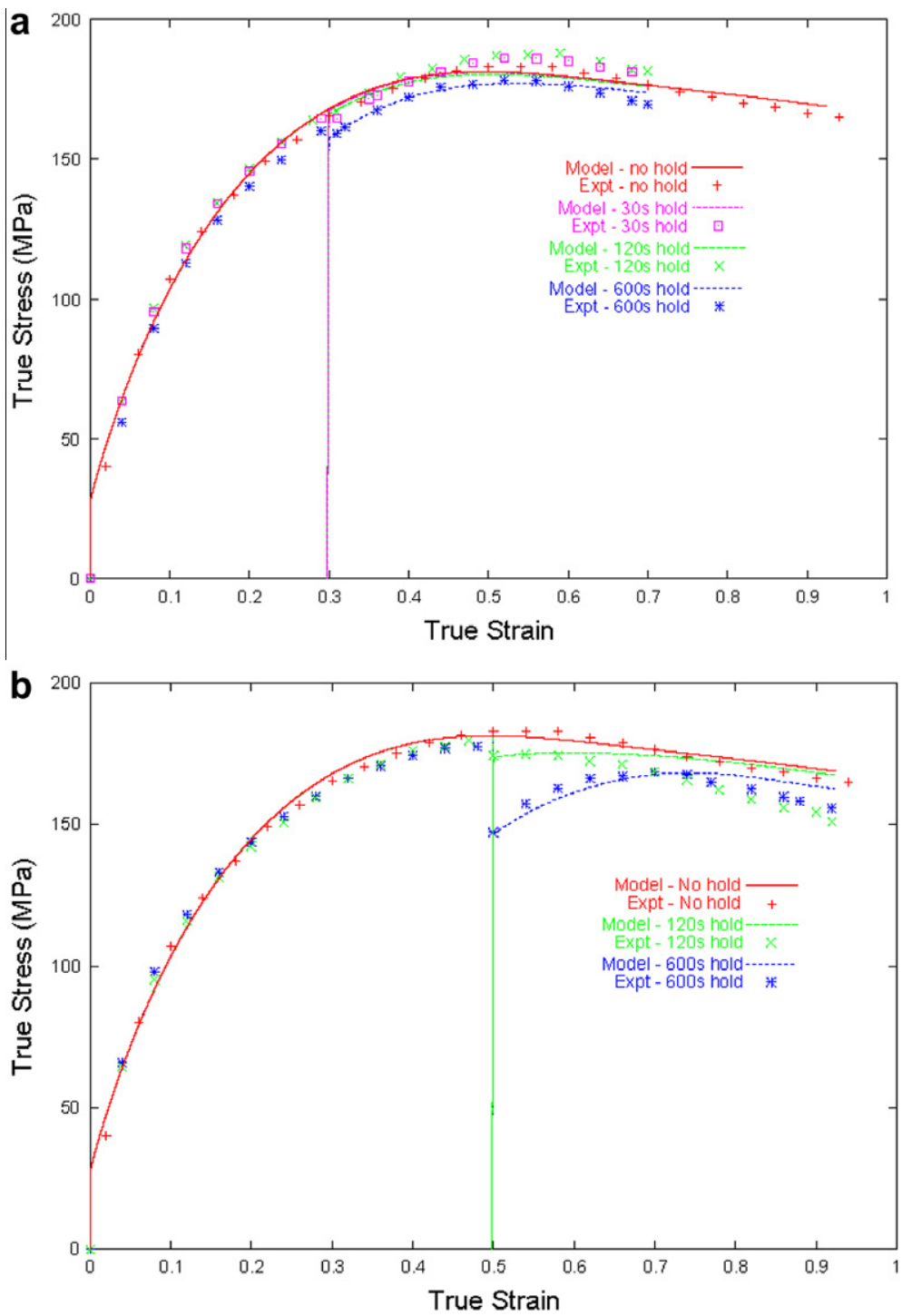

Fig. 12. Validation of the model using the optimized parameters to predict multi-stage loading in which specimens are compressed at $0.0004 / \mathrm{s}$ to strain levels of (a) 0.3 and (b) 0.5 at $269{ }^{\circ} \mathrm{C}$, held for times ranging from $30 \mathrm{~s}$ to $600 \mathrm{~s}$, and then further compressed at $0.0004 / \mathrm{s}$ to strain levels of (a) 0.7 and (b) 0.92 , respectively. The model predicts both the static recrystallization that occurs between loading stages as well as the dynamic recrystallization occurring during the second loading stage.

material initially hardens, but at higher strains dynamic recrystallization occurs and decreases the yield strength. The model accurately predicts both the static recrystallization that occurs between loading stages as well as the hardening and subsequent dynamic recrystallization occurring during the second loading stage.

In Fig. 12(b), the first loading stage is continues to a strain of 0.5 before deformation is ceased. The higher rate of static recrystallization that results leads to a more substantial drop in the yield stress, as observed upon reloading for the second stage. Once again, the model accurately predicts the amount of softening that occurs during the various hold times. It also predicts subsequent dynamic recrystallization during the second loading stage, although it slightly underpredicts the resulting softening evident in the experimental data.

For the two-stage test strained to 0.5 , then held for $600 \mathrm{~s}$ and reloaded, the evolution of the average isotropic hardening variable as well as the breakdown by volume fraction is shown in Fig. 13 as a function of time. The recrystallization kinetics and the isotropic hardening variables in each volume fraction are shown in Figs. 14 and 15, respectively. The curves look similar to single-stage tests, but with a 600-s period of static recrystallization with an accompanying drop in the contribution to the average isotropic hardening variable from the unrecrystallized volume fraction. Note that the isotropic hardening variable $\kappa_{X_{1}-X_{2}}$ in the volume fraction $X_{1}-X_{2}$ decreases during static recrystallization due to newly recrystallized material, 


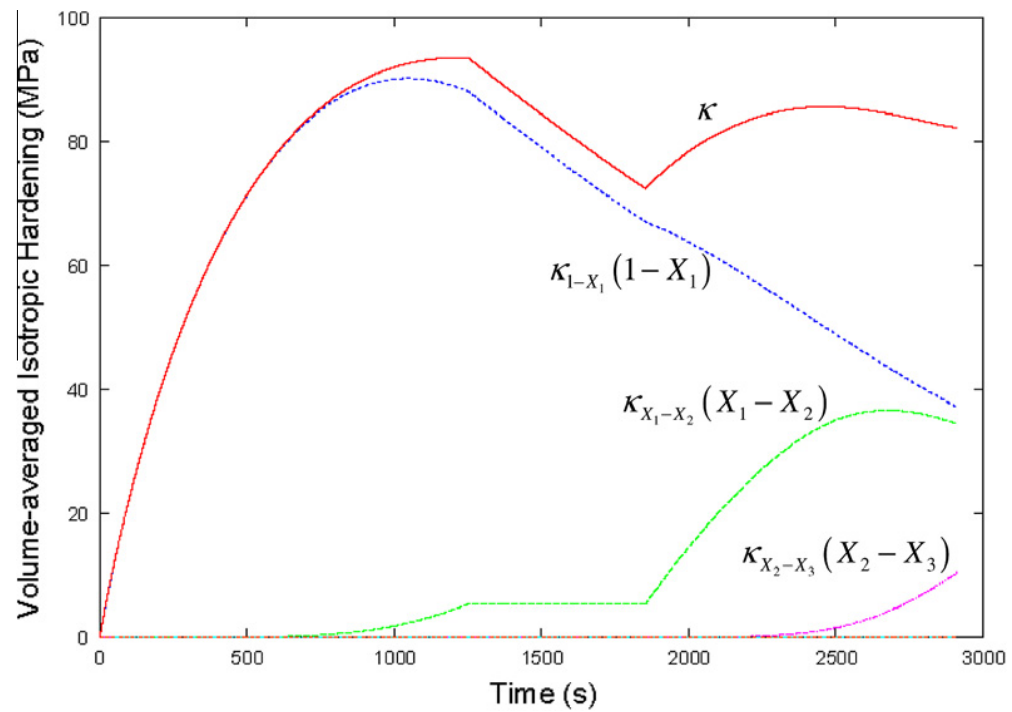

Fig. 13. The contributions to the average isotropic hardening variable from each volume fraction for a strain rate of $0.0004 / \mathrm{s}$ at $269{ }^{\circ} \mathrm{C}$.

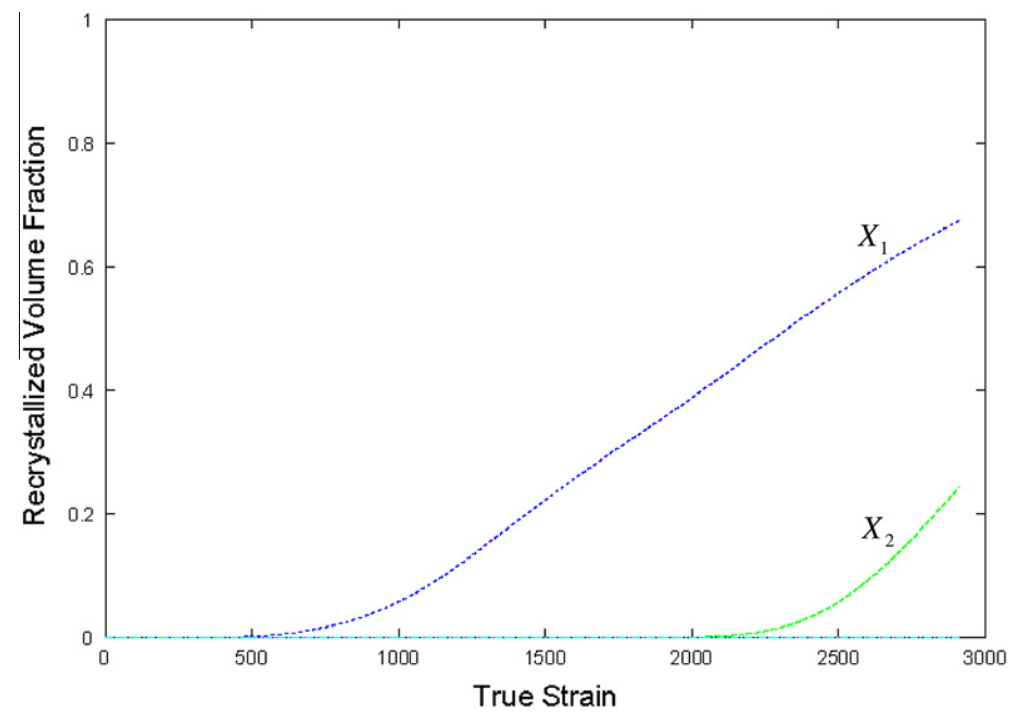

Fig. 14. Evolution of the recrystallization cycles for a strain rate of $0.0004 / \mathrm{s}$ at $269{ }^{\circ} \mathrm{C}$.

but the contribution $\kappa_{X_{1}-X_{2}}\left(X_{1}-X_{2}\right)$ of that volume fraction to the average isotropic hardening variable remains the same (Fig. 13) due to the corresponding increase in $X_{1}-X_{2}$.

Fig. 16 shows the results for two-stage tests that involve a temperature change in addition to a hold time between loadings. Specimens are initially loaded at room temperature to a strain of 0.5 , then heated to $269{ }^{\circ} \mathrm{C}$, held in the furnace for hold times between 1.5 and $20 \mathrm{~min}$, and then reloaded at $269^{\circ} \mathrm{C}$. When the specimen hold time is limited to 1.5 min, the initial experimental response during subsequent loading at $269^{\circ} \mathrm{C}$ is softening. For the 20 -min hold time, the second stage shows a much lower yield stress and its initial behavior is hardening. For both cases, dynamic recrystallization is evident at larger strains. The model predictions exhibit similar behavior, with substantially more static recrystallization evident for the larger hold time, and initial slopes indicating softening for the short hold time and hardening for the long hold time. The model does not accurately capture the initial yield stress for the second stage, but accurately matches the stress and dynamic recrystallization at larger strains. The predicted response for this model is a substantial improvement over the response of the Johnson-Cook, Mechanical Threshold Stress, and BCJ-SNL models that do not incorporate recrystallization for the same loading conditions, as shown in Tanner et al. (1999). 


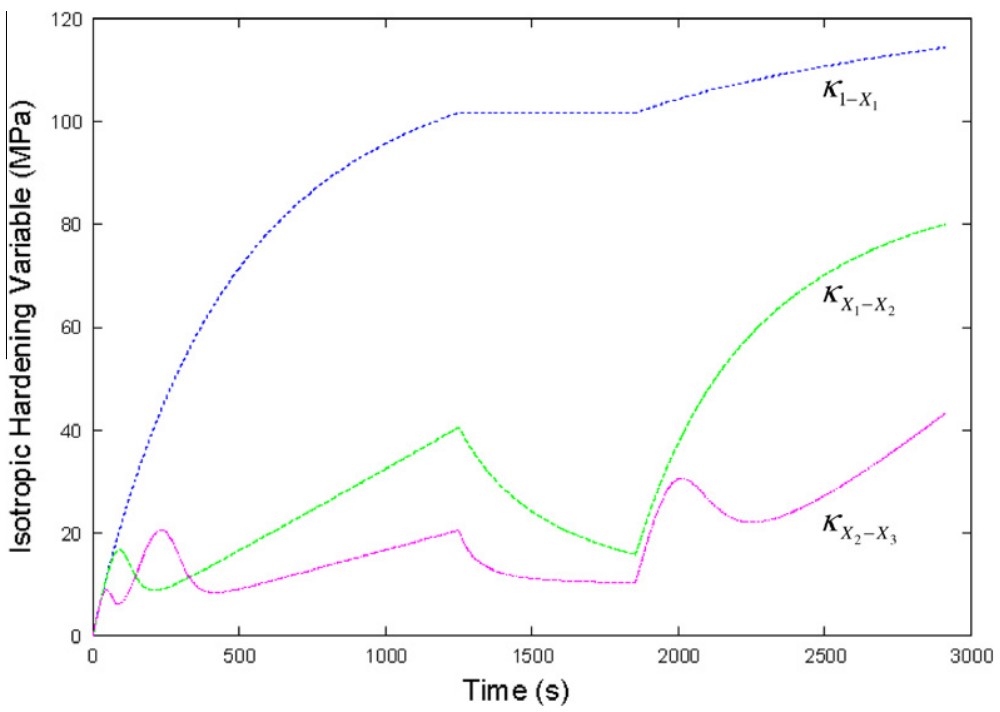

Fig. 15. The isotropic hardening variable in each volume fraction for a strain rate of $0.0004 / \mathrm{s}$ at $269{ }^{\circ} \mathrm{C}$.

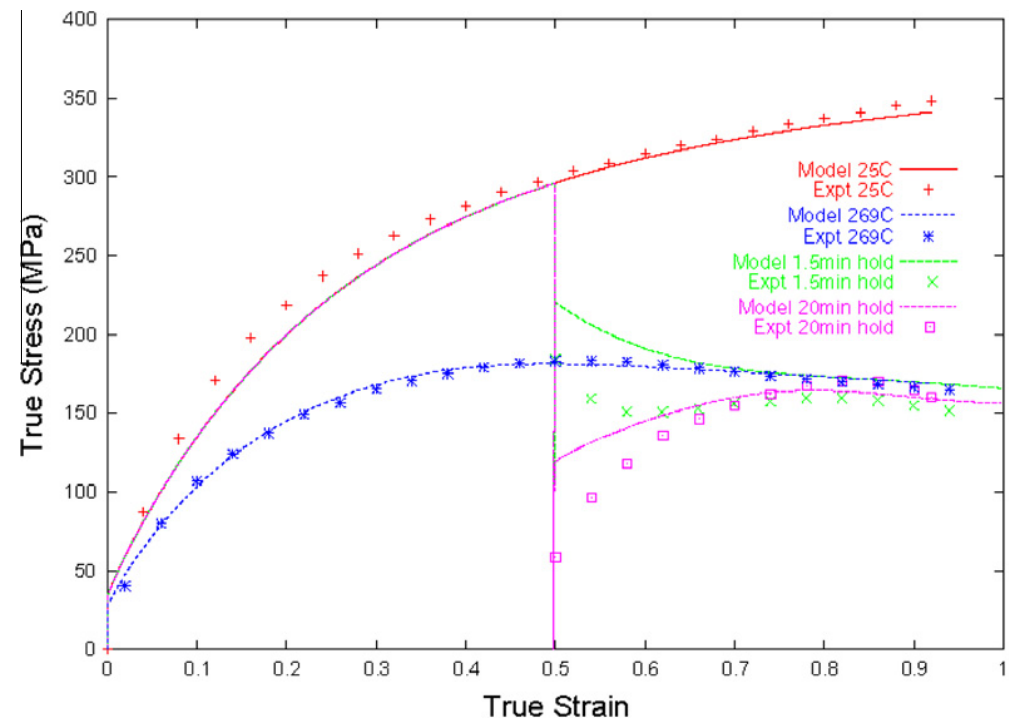

Fig. 16. Model prediction compared to multi-stage experiments in which specimens are compressed at $0.0004 / \mathrm{s}$ and $25{ }^{\circ} \mathrm{C}$ to a strain of 0.5 , then heated to $269^{\circ} \mathrm{C}$, held for times ranging from 1.5 to $20 \mathrm{~min}$, and then further compressed at $0.0004 / \mathrm{s}$ to a strain of 0.94 .

\section{Conclusions}

A novel constitutive model is presented that is capable of predicting both static and dynamic recrystallization. The model includes internal state variables representing statistically stored dislocations and geometrically necessary dislocations. Recrystallization cycles evolve based on the stored energy in the dislocation structure. A rule of mixtures is used to determine the average values of the state variables. The model is able to match both single and multiple peak dynamic recrystallization. Single peak behavior occurs when the cycles of recrystallization overlap one another significantly. At higher temperatures and slower strain rates, the recrystallization cycles do not overlap as much, leading to multiple distinct peaks in the stress-strain curves. The theory is also shown to be capable of modeling multiple stages of loading, with multiple cycles of static and dynamic recrystallization occurring. The model was validated by optimizing parameters to single-stage compression data, then using the same parameter set to predict multiple-stage responses.

To keep the formulation less complex, only scalar state variables were presented. Using a single scalar variable to represent geometrically necessary boundaries is an over-simplification that could be corrected in the future. While Hughes et al. (1997) and Hughes and Hansen (2000) have shown that the average spacing of the geometrically necessary boundaries is 
inversely proportional to the average misorientation angle across the boundaries during deformation, this does not hold true during recrystallization. For example, during recrystallization, subgrains grow at the expense of their neighbors, which increases the average spacing, but does not necessarily decrease the average misorientation angle. In future work, the scalar misorientation variable could be replaced with a tensorial variable like that used in Bammann (2001). Also, texture could be included in the model through the use of a structure tensor that would lead to an anisotropic plastic response (see Regueiro et al., 2002).

In a future paper, work will be presented in which the recrystallization parameters are fit to microstructural experimental data, and accurate final strength predictions are then made for forged material.

\section{Acknowledgements}

This work was performed at Sandia National Laboratories. Sandia is a multiprogram laboratory operated by Sandia Corporation, a Lockheed Martin Company, for the United States Department of Energy under contract DEAC04-94AL85000.

\section{References}

Ashby, M.F., 1970. The deformation of plastically non-homogeneous materials. Philos. Mag. 21, 399-424.

Avrami, M.J., 1939. Kinetics of phase change. Chem. Phys. 7, 1103-1109.

Bailey, J.E., Hirsch, P.B., 1962. The recrystallization process in some polycrystalline metals. Proc. Roy. Soc. A267, 11-30.

Bammann, D.J., 2001. A model of crystal plasticity containing a natural length scale. Mater. Sci. Eng. A309-310, 406-410.

Bammann, D.J., Chiesa, M.L., Johnson, G.C., 1995. A state variable model for temperature and strain rate dependent metals. In: Rajendran, A.M., Batra, R.C.

(Eds.), Constitutive Laws: Experiments and Numerical Implementation. CIMNE, pp. 84-97

Bammann, D.J., Chiesa, M.L., Johnson, G.C., 1996. Modeling large deformation and failure in manufacturing processes. In: Tatsumi, T., Watanabe, E., Kambe, T. (Eds.), Theoretical and Applied Mechanics. ICTAM, pp. 359-376.

Busso, E.P., 1998. A continuum theory for dynamic recrystallization with microstructure-related length scales. Int. J. Plant 14, 319-353.

Cahn, J.W., Hagel, W., 1960. Theory of the pearlite reaction. In: Zackay, Z.D., Aaronson, H.I. (Eds.), Decomposition of Austenite by Diffusional Processes. Interscience, pp. 131-196.

Casey, J.C., Naghdi, P.M., 1992. A prescription for the identification of finite plastic strain. Int. J. Eng. Sci. 30 (10), 1257-1278.

Chen, S.P., Todd, I., van der Zwaag, S., 2002. Modeling the kinetics of grain-boundary-nucleated recrystallization processes after cold deformation. Metall. Mater. Trans. A 33 (3), 529-537.

Chiesa, M.L., Brown, A.A., Antoun, B.R., Ostien, J.T., Regueiro, R.A., Bammann, D.J., Yang, N.Y., 2004. Prediction of final material state in multi-stage forging processes. AIP Conf. Proc. (712), 510-515, pt. 1.

Coleman, B.D., Gurtin, M.E., 1967. Thermodynamics with internal state variables. J. Chem. Phys. 47, 597-613.

Coleman, B.D., Noll, W., 1963. The thermodynamics of elastic materials with heat conduction and viscosity. Arch. Ration. Mech. Anal. 13, 167-178.

Doherty, R.D., 2005. Primary recrystallization. In: Cahn, R.W. et al. (Eds.), Encyclopedia of Materials: Science and Technology. Elsevier, pp. 7847-7850.

Doherty, R.D., Hughes, D.A., Humphreys, F.J., Jonas, J.J., Jensen, D.J., Kassner, M.E., King, W.E., McNelley, T.R., McQueen, H.J., Rollett, A.D., 1997. Current issues in recrystallization: a review. Mater. Sci. Eng. A238, 219-274.

Estrin, Y., Mecking, H., 1984. A unified phenomenological description of work hardening and creep based on one-parameter models. Acta Metall. 32 (1), 5770.

Fan, X.G., Yang, H., 2011. Internal-state-variable based self-consistent constitutive modeling for hot working of two-phase titanium alloys coupling microstructure evolution. Int. J. Plant 27, 1833-1852.

Fox, N., 1968. On the continuum theories of dislocations and plasticity. Q. J. Mech. Appl. Math. 21, 67-75.

Frost, H.J., Ashby, M.F., 1982. Deformation-Mechanism Maps. Pergamon Press, New York.

Furu, T., Orsund, R., Nes, E., 1995. Subgrain growth in heavily deformed aluminium - experimental investigation and modelling treatment. Acta Metall. Mater. 43 (6), 2209-2232.

Gottstein, G., 2001. Physikalische Grundlagen der Metallkunde, second ed. Springer, Berlin/Heidelberg, Germany.

Haessner, F. (Ed.), 1978. Recrystallization of Metallic Materials, second ed. Rieder-Verlag, Stuttgart.

Holm, E.A., Miodownik, M.A., Rollett, A.D., 2003. On abnormal subgrain growth and the origin of recrystallization nuclei. Acta Mater. 51, 2701-2716.

Huang, Y., Humphreys, F.J., 1999. Measurements of grain boundary mobility during recrystallization in a single-phase aluminium alloy. Acta Mater. 47, 2259-2271.

Huang, Y., Humphreys, F.J., 2000. Subgrain growth and low angle boundary mobility in aluminum crystals of orientation $\{110\}\langle 001\rangle$. Acta Mater. 48, 20172030.

Hughes, D.A., Hansen, N., 2000. Microstructure and strength of nickel at large strains. Acta Mater. 48, 2985-3004.

Hughes, D.A., Liu, Q., Chrzan, C.C., Hansen, N., 1997. Scaling of microstructural parameters: misorientation of deformation-induced boundaries. Acta Mater. $45(1), 105-112$.

Humphreys, F.J., Hatherly, M., 1995. Recrystallization and Related Annealing Phenomena. Pergamon Press, Oxford.

Johnson, W.A., Mehl, R.F., 1939. Reaction kinetics in processes of nucleation and growth. Trans. Am. Inst. Min. Eng. 135, 416-430.

Kocks, U.F., Mecking, H., 1979. A mechanism for static and dynamic recovery. In: Haasen, P., Gerold, V., Kostorz, G. (Eds.), Strength of Metals and Alloys. Pergamon Press, Oxford, pp. 345-350.

Kocks, U.F., Argon, A.S., Ashby, M.F., 1975. The thermodynamics and kinetics of slip. Prog. Mater. Sci. 19, 1-291.

Kok, S., Beaudoin, A.J., Tortorelli, D.A., 2002. On the development of stage IV hardening using a model based on the mechanical threshold. Acta Mater. 50, $1653-1667$

Kolmogorov, A.E., 1937. On the statistical theory of metal crystallization. Izv. Akad. Nauk SSSR, Ser. Mat. 3, 355-359.

Kröner, E., 1960. Allgemeine kontinuumstheorie derivative versetzungen and eigenspannungen. Arch. Ration. Mech. Anal. 4, $273-334$.

Lathrop, J.F., 1997. BFIT - a program to analyze and fit the BCJ model parameters to experimental data - tutorial and user's guide. Sandia National Laboratories report SAND97-8218.

Ledbetter, H.M., Naimon, E.R., 1974. Elastic properties of metals and alloys. II. Copper. J. Phys. Chem. Ref. Data 3, 897-935.

Lee, E.H., 1969. Elastic-plastic deformation at finite strains. ASME J. Appl. Mech. 36, 1-6.

Lee, E.H., Liu, D.T., 1967. Finite-strain elastic-plastic theory with application to plane-wave analysis. J. Appl. Phys. 38, 19-27.

Luton, M.J., Sellars, C.M., 1969. Dynamic recrystallization in nickel and nickel-iron alloys during high temperature deformation. Acta Metall. 17, $1033-1043$.

Mandel, J., 1974. Thermodynamics and plasticity. In: Delgado, J.J. et al. (Eds.), Foundations of Continuum Thermodynamics. Macmillan, New York, pp. 283304.

Manonukul, A., Dunne, F.P.E., 1999. A model for the initiation of dynamic recrystallization in two-phase materials. Phil. Mag. 79, 113-132.

Marin, E.B., Bammann, D.J., Regueiro, R.A., Johnson, G.C., 2006. On the formulation, parameter identification and numerical integration of the EMMI model: plasticity and isotropic damage. Sandia National Laboratories report SAND2006-0200. 
McQueen, H.J., Jonas, J.J., 1975. Recovery and recrystallization during high temperature deformation. In: Arsenault, R.J. (Ed.), Plastic Deformation of Materials, vol. 6. Academic Press, New York, pp. 393-493.

Pietrzyk, M., 2002. Through-process modeling of microstructure evolution in hot forming of steels. J. Mater. Proc. Tech., $53-62$.

Prantil, V.C., Jenkins, J.T., Dawson, P.R., 1993. An analysis of texture and plastic spin for planar polycrystals. J. Mech. Phys. Solids 41, $1357-1382$.

Regueiro, R.A., Bammann, D.J., Marin, E.B., Garikipati, K., 2002. A nonlocal phenomenological anisotropic finite deformation plasticity model accounting for dislocation defects. J. Eng. Mater. Tech. 124, 380-387.

Roberts, W., Ahlblom, B., 1978. A nucleation criterion for dynamic recrystallization during hot working. Acta Metall. $26,801-813$.

Rollett A.D. Holm E.A., 1996. Abnormal grain growth-the origin of recrystallization nuclei? In: McNelley, T.R. (Ed.), Rex-96. MRS, pp. 31-42.

Roucoules, C., Pietrzyk, M., Hodgson, P.D., 2003. Analysis of work hardening and recrystallization during hot working of steel using a statistically based internal variable model. Mat. Sci. Eng. A339, 1-9.

Sakai, T., Jonas, J.J., 1984. Dynamic recrystallization: mechanical and microstructural considerations. Acta Metall. 32, 189-209.

Sandstrom, R., Lagneborg, R., 1975. A model for hot working occurring by recrystallization. Acta Metall. 23, $387-398$.

Speich G.R. Fisher R.M., 1966. Recrystallization in rapidly heated $31 / 4 \%$ silicon iron. In Margolin, H. (Ed.), Recrystallization, grain growth and textures. ASM, pp. 563-598.

Svyetlichnyy, D.S., 2007. A coupled model of flow stress and microstructure evolution. NUMIFORM'07, Mater. Proc. Design, pp. 1307-1312.

Tanner, A.B., McDowell, D.L., 1999. Deformation, temperature and strain rate sequence experiments on OFHC Cu. Int. J. Plant 15 (4), $375-399$.

Tanner, A.B., McGinty, R.D., McDowell, D.L., 1999. Modeling temperature and strain rate history effects in OFHC Cu. Int. J. Plant 15 (4), $575-603$.

Taylor, G.I., 1934. The mechanism of plastic deformation of crystals. Part I. Theoretical. Proc. R. Soc. London, Ser. A. A145, 362-387.

Wray, P.J., 1975. Onset of recrystallization during the tensile deformation of austenitic iron at intermediate strain rates. Metall. Trans. A 6 (6), 1197-1203. 